\title{
Intestine-Liver Axis On-Chip Reveals the Intestinal Protective Role on Hepatic Damage by Emulating Ethanol First-Pass Metabolism
}

\author{
Vincenza De Gregorio 1,2, Mariarosaria Telesco ${ }^{1}$, Brunella Corrado ${ }^{1}$, Valerio Rosiello ${ }^{2}$, \\ Francesco Urciuolo ${ }^{2}$, Paolo A. Netti ${ }^{1,2,3}$ and Giorgia Imparato ${ }^{1 *}$ \\ ${ }^{1}$ Center for Advanced Biomaterials for HealthCare@CRIB, Istituto Italiano di Tecnologia, Naples, Italy, ${ }^{2}$ Interdisciplinary \\ Research Centre on Biomaterials (CRIB), University of Naples Federico II, Naples, Italy, ${ }^{3}$ Department of Chemical, Materials \\ and Industrial Production Engineering (DICMAPI) University of Naples Federico II, Naples, Italy
}

OPEN ACCESS

Edited by:

María García-Díaz,

Institute for Bioengineering of

Catalonia (IBEC), Spain

Reviewed by:

Tiziano Verri,

University of Salento, Italy

Salman Khetani,

University of Illinois at Chicago,

United States

Christopher B. Forsyth,

Rush University, United States

${ }^{*}$ Correspondence:

Giorgia Imparato

giorgia.imparato@iit.it

Specialty section

This article was submitted to Tissue Engineering and Regenerative Medicine,

a section of the journal Frontiers in Bioengineering and Biotechnology

Received: 28 November 2019 Accepted: 18 February 2020

Published: 17 March 2020

Citation:

De Gregorio V, Telesco M, Corrado B,

Rosiello V, Urciuolo F, Netti PA and Imparato G (2020) Intestine-Liver Axis

On-Chip Reveals the Intestinal

Protective Role on Hepatic Damage

by Emulating Ethanol

First-Pass Metabolism

Front. Bioeng. Biotechnol. 8:163. doi: 10.3389/fbioe.2020.00163
Intestine-Liver-on-chip systems can be useful to predict oral drug administration and first-pass metabolism in vitro in order to partly replace the animal model. While organ-on-chip technology can count on sophisticated micro-physiological devices, the engineered organs still remain artificial surrogates of the native counterparts. Here, we used a bottom-up tissue engineering strategy to build-up physiologically functional 3D Human Intestine Model (3D-HIM) as well as 3D Liver-microtissues (HepG2- $\mu$ TPs) in vitro and designed a microfluidic Intestine-Liver-On-Chip (InLiver-OC) to emulate first-pass mechanism occurring in vivo. Our results highlight the ethanol-induced 3D-HIM hyper-permeability and stromal injury, the intestinal prevention on the liver injury, as well as the synergic contribution of the two 3D tissue models on the release of metabolic enzymes after high amount of ethanol administration.

\footnotetext{
Keywords: first-pass metabolism of ethanol (Et-OH), intestine-liver-on-chip, bottom-up tissue engineering approach, endogenous ECM, 3D tissue
}

\section{INTRODUCTION}

Orally administered drugs and chemicals are primarily metabolized by the gastrointestinal (GI) tract and liver (de Sousa and Bernkop-Schnürch, 2014). The xenobiotics and their metabolites interact with the small intestine, causing damage, and a fraction of these is transported across the gut epithelium and absorbed into the bloodstream before reaching the liver by the portal vein (Maurice et al., 2013). Therefore, there is a need to understand how such compounds may affect function at the organ level. Traditionally, testing the toxic effects of chemicals or drugs has relied on large-scale animal studies (Council, 2007). In addition to being extremely expensive, the data obtained with animal models often cannot be extrapolated to humans (Esch et al., 2011). In this perspective, there is a need to design in vitro organotypic cultures to investigate toxicity. In the last few years, in vitro models of the GI tract (Huh et al., 2011; Bhatia and Ingber, 2014) and liver (Santaguida et al., 2006; Inamdar and Borenstein, 2011) have been developed individually, but single-organ systems lack the complex intercellular and inter-organ communication that occurs in vivo and therefore are unable to properly model the enterohepatic circulation and first-pass metabolism that have been shown to be critical for assessing drug and xenobiotics metabolism. For this reason, recently, multi-compartment microfluidic-based devices (organs-on-a-chip) have been proposed to increase safety and the efficacy of the drug development process. These platforms 
consist of microfluidic cell culture devices, fabricated by means of microfabrication methods, which contain continuously perfused chambers inhabited by living cells arranged to simulate tissue- and organ-level physiology. By recapitulating the multicellular architectures, tissue-tissue interfaces, physicochemical microenvironments, and vascular perfusion of the body, these devices exceed the static conventional $2 \mathrm{D}$ or $3 \mathrm{D}$ culture systems (Esch et al., 2012). In an attempt to recapitulate the integration of the GI tract and the liver mimicking the first-pass metabolism, several in vitro models have been established. Choe et al. have recently developed a microfluidic device that consists of $2 \mathrm{D}$ cell models seeded into two separate compartments for gut epithelial cells (Caco-2) and liver cells (HepG2), respectively, designed so that drugs go through a sequential absorption in the gut chamber and metabolic reaction in the liver chamber (Choe et al., 2017). In another work, 3D gut model and Hepg2 layer were integrated into microfluidic device to recapitulate the complex process of absorption and metabolism of digested lipids on in vitro model of hepatic steatosis (Lee and Sung, 2018). Another noteworthy model built up by Shuler's group is based on pumpless modular GI-liver model to co-culture human intestinal cells and 3D hepatic cells in order to replicate human GI-liver physiology (Chen et al., 2018). On the other hand, van Midwoud et al. developed a microfluidic system for the sequential perfusion of rat intestinal and liver slices for metabolism studies (van Midwoud et al., 2010). However, this approach cannot be easily translated to the use of human tissue slices, whose viability dramatically decreases over a few days of culture, making this tissue model infeasible for organ-on-chip purposes. Other studies are based on first-pass metabolism of orally administered drugs by using multi-compartment pharmacokinetic models. In particular, Mahler et al. developed a microfluidic biochip in which HT-29 goblet-like cells are included in the GI-tract compartment lining the Caco- 2 cell layer with mucous granules, in order to demonstrate absorption, distribution, metabolism, and toxicity (ADME-Tox) of acetaminophen (APAP) (Mahler et al., 2009). Also, Prot et al. analyzed the metabolism of paracetamol through a microfluidic device, which comprises the Caco- 2 cells cultivated on a conventional cell culture insert introduced in a bioreactor and the liver cells modeled by HepG2/C3a isolated from rat/human primary hepatocytes. Their experiments were also supported by a mathematical model to estimate intrinsic in vitro parameters and to predict in vivo processes (Prot et al., 2014). In a recent work, an integrated gutliver microphysiological systems was used to elucidate the intertissue cross-talk under inflammatory conditions, focusing on the long-term co-culture into the device to reproduce pathological conditions (Chen et al., 2017). However, even if these systems (Esch et al., 2014) provide important insights into the molecular mechanism of GI tract metabolism, they include over-simplified cell mono or co-culture models, thus failing in the replication of the 3D physiological ECM microenvironment with its dynamic complexity. Our studies have recently demonstrated the crucial role of the native ECM remodeling on the intestine adsorptive and metabolic function as well as the ECM involvement in the intestinal mucosal inflammation (De Gregorio et al., 2019). With the perspective of elucidating the inter-organs crosstalk with a more physiological functional 3D tissues, we reported a development of a novel Intestine-Liver-on-Chip (InLiver-OC) consisting of two directly interconnected chambers (Intestine-compartment and Liver-compartment) that enable the culture of a 3D Human Intestine Model (3D-HIM) as well as $3 \mathrm{D}$ liver microtissues (HepG2- $\mu \mathrm{TPs}$ ) in order to simulate the physiological mechanism of the first-pass metabolism of orally-ingested compounds. Bottom-up approach was used to fabricate the 3D-HIM provided with an endogenous ECM (Imparato et al., 2013) and HepG2- $\mu$ TPs, as previously reported (Corrado et al., 2019). The combination of such physiological relevant $3 \mathrm{D}$ models with microfluidic technology allowed us to investigate the protective role of the intestine on liver injury, analyzing the 3D-HIM hyper-permeability as well as the intestinal stroma remodeling. In addition, in this system, the integrated InLiver-OC was used to study the synergic contribution of the two $3 \mathrm{D}$ tissue models on the release of metabolic enzymes after high amount of ethanol (Et-OH) administration as exogenous stimulus mimicking the "binge drinking." This multifunctional system has a broad applicability toward advancing fundamental understanding of human pathophysiology and drug development processes.

\section{MATERIALS AND METHODS}

\section{Cell Type}

For 3D-HIM production, human intestinal myofibroblasts (H-InMyoFib) were purchased from Lonza and cultured in SMGMTM-2 BulletKitTM medium (Lonza). H-InMyoFibs at 35 passages were used. Human intestinal epithelial cells (Caco-2) were provided by American Type Culture Collection (ATCC) and were cultured in Dulbecco Modified Eagle Medium (DMEM, Microtech) with $10 \%$ of fetal bovine serum (FBS, Microtech), $100 \mu \mathrm{g} \mathrm{mL}^{-1} \mathrm{~L}$-glutamine, $100 \mathrm{U} \mathrm{mL}^{-1}$ penicillin/streptomycin. For HepG2- $\mu$ TPs production, hepatocellular carcinoma cells (HepG2) cells were purchased by ATCC and were cultured in Minimum Essential Medium Earle's Salt (MEM, Microtech), containing $10 \%$ fetal bovine serum, $100 \mu \mathrm{g} \mathrm{mL}^{-1} \mathrm{~L}$-glutamine, $100 \mathrm{U} \mathrm{mL} \mathrm{m}^{-1}$ penicillin/streptomycin, $0.1 \mathrm{mM}$ Non-Essential Amino Acid and $0.1 \mathrm{mM}$ Sodium pyruvate. Cells were incubated at $37^{\circ} \mathrm{C}$ in a humidified atmosphere with $5 \% \mathrm{CO}_{2}$.

\section{D Human Intestine Model (3D-HIM) Production}

Gelatine porous microscaffolds (GPMs) (75-150 $\mu \mathrm{m}$ diameter) were made-up by a slightly adapted double emulsion method $(\mathrm{O} / \mathrm{W} / \mathrm{O})$ and stabilized with glyceraldehyde at $4 \%$ as previously described (Imparato et al., 2013). Briefly, $10 \mathrm{~mL}$ of water containing TWEEN $85(6 \% \mathrm{w} / \mathrm{v})$ were used to dissolve the gelatin powder (type B, Mw 176,654 Da) (Sigma Aldrich) at $60^{\circ} \mathrm{C}$. Then, an oil in water $(\mathrm{O} / \mathrm{W})$ emulsion was produced by adding a solution of Toluene and SPAN $85(3 \% \mathrm{w} / \mathrm{v})$ to the aqueous gelatin solution $(8 \% \mathrm{w} / \mathrm{v})$ (Sigma Aldrich). To obtain gelatin micro-beads, $30 \mathrm{~mL}$ of additional toluene were added $(\mathrm{O} / \mathrm{W} / \mathrm{O})$ and cooled to $5^{\circ} \mathrm{C}$. At last, to obtain the toluene extraction and gelatin micro-beads stabilization, ethanol $(20 \mathrm{~mL})$ was poured. Dried GPMs were sterilized with ethanol, 
washed with PBS, and then inoculated $(2 \mathrm{mg} / \mathrm{mL}$ of GPMs; 5 $\times 10^{3}$ beads $\left./ \mathrm{mg}\right)$ with $\mathrm{H}$-InMyoFibs $\left(1.5 \times 10^{5} \mathrm{cell} / \mathrm{mL}\right)$ and cultured in a dynamic conditions in spinner flask bioreactor (CELLSPIN, $250 \mathrm{ml}$, Integra Biosciences) to obtain Human Intestine- $\mu$ TPs (HI- $\mu$ TPs) (De Gregorio et al., 2018a). In order to promote H-InMyoFibs adhesion, spinner flask operated with and intermittent stirring for $6 \mathrm{~h}$ post inoculation ( $5 \mathrm{~min}$ shaking, $40 \mathrm{~min}$ stationary) and continuous stirring at $20 \mathrm{rpm}$ was set for 10 days. Spinner flask bioreactor operated in the cell incubator at $37^{\circ} \mathrm{C}$ and $5 \% \mathrm{CO}_{2}$; the media replacement occurred every 2 days, and 2-O-alpha-D-Glucopyranosyl-L-ascorbic Acid $0.5 \mathrm{mM}$ (TCI Europe) was added at each culture media change. After 10 days of spinner flask bioreactor culture, HI- $\mu$ TPs were transferred into a maturation chamber bioreactor where HI$\mu$ TPs assembling took place forming disc-shaped ( $1 \mathrm{~mm}$ thick, $6 \mathrm{~mm}$ diameter) 3D Intestinal stroma (3D-ISs) (De Gregorio et al., 2018a). The maturation chamber was inserted into a spinner flask programmed at $60 \mathrm{rpm}$. After 2 weeks of culture, the maturation chamber was opened, the 3D-ISs were withdrawn and washed 2-folds with PBS solution, then transferred into a transwell insert (6.5 mm diameter; Corning), and left to dry for 5 min under laminar hood. Further, Caco- 2 cells suspension $(2 \times$ $10^{5}$ cells in $50 \mu \mathrm{L}$ ) was seeded on each 3D-IS in order to produce the intestinal epithelium. The samples were incubated for about $2 \mathrm{~h}$ at $37^{\circ} \mathrm{C}, 5 \% \mathrm{CO}_{2}$ to permit Caco- 2 cells adhesion onto the $3 \mathrm{D}$ IS apical surface. Further, submerged culture was performed by adding $200 \mu \mathrm{L}$ of DMEM on the apical side of the 3D-IS and 600 $\mu \mathrm{L}$ of DMEM in the baso-lateral side for 7 days. Then, to induce the epithelial cells polarization and differentiation, the samples were cultured for 2 weeks in an air-liquid interface culture. Three times per week the culture medium was replaced. At the end of the experiment, the full-thickness intestinal equivalents (3DHIMs) composed by 3D-IS overlapped by intestinal epithelium were taken from the transwell insert for further investigation or were loaded into the Intestine chamber of the Intestine-on-Chip (In-OC) or Intestine-Liver-on-Chip (InLiver-OC).

\section{HepG2- $\mu$ TPs Development}

HepG2 cells were cultured on GPMs in spinner flasks (Integra), as previously reported with slight modifications (Corrado et al., 2019). Briefly, $35 \mathrm{mg}$ of GPMs were loaded with $5.25 \times 10^{6}$ cells (30 cell/GPM ratio). To promote cell seeding on GPMs an intermittent stirring regime $(30 \mathrm{~min}$ at $0 \mathrm{rpm}, 5 \mathrm{~min}$ at 30 $\mathrm{rpm}$ ) was applied for $24 \mathrm{~h}$. Afterwards, the stirring speed was kept at a continuous $20 \mathrm{rpm}$ for up to 7 days. Culture medium was changed three times per week. All cultures were maintained at $37^{\circ} \mathrm{C}$ in a humidified $5 \% \mathrm{CO}_{2}$ incubator for $6-7$ days, and then HepG2- $\mu$ TPs were loaded into microfluidic Liver-On-Chip (Liver-OC) or into InLiver-OC.

\section{Microfluidic Device Fabrication}

The microfluidic InLiver-OC device was fabricated by a rapid prototyping procedure. The PMMA master mold was designed by AutoCAD and carved with micromilling machine (Minithech CNC Mini-Mill) making a relief positive geometry. A mixture of PDMS pre-polymer and curing agent 10:1 (w/w) was prepared, degassed under vacuum for $20 \mathrm{~min}$ to remove air bubbles, and then poured on PMMA master. The set-up was incubated at $80^{\circ} \mathrm{C}$ for $60 \mathrm{~min}$, then peeled off from master molds. The device consists of two compartments, the intestine compartment (Intestine ${ }_{\mathrm{c}}$ ) and the liver compartment ( Liver $_{\mathrm{c}}$ ), which are connected by a central microchannel. The Intestine ${ }_{c}$ was designed with a central microchannel $(1.2 \mathrm{~mm}$ wide $\times$ $40 \mathrm{~mm}$ long $\times 0.6 \mathrm{~mm}$ high), which transported the medium into an intestine chamber (9.5 mm diameter $\times 5 \mathrm{~mm}$ high). The Liver $_{\mathrm{c}}$ communicated with the Intestine ${ }_{c}$ by a central microchannel, which was separated from three parallels chambers $(0.5 \mathrm{~mm}$ wide $\times 0.6 \mathrm{~mm}$ high $\times 1 \mathrm{~mm}$ long) by micro-pillars $(0.100 \mathrm{~mm}$ diameter $\times 0.09 \mathrm{~mm}$ pillar interspace). A collection channel $(0.5 \mathrm{~mm}$ wide $\times 0.3$ high $)$ was used to collect tissue supernatants. Culture medium inlet and outlet, as well as inlet for HepG2$\mu$ TPs loading in the Liver $_{c}$, were punched with a $2.5 \mathrm{~mm}$ biopsy punch (DifaCooper), while intestine chamber of the Intestine ${ }_{c}$ was punched using a 9.5-mm puncher (Am-Tech) (Figure S1). Then, a transwell insert was placed into the $9.5-\mathrm{mm}$ holes of the biochip of the Intestine . To ensure the selective transport of fluid through the 3D-HIM, a silicon gasket and a PMMA cylinder were fabricated. In detail, PDMS gasket was fabricated by punching 1-mm-thick PDMS layer, first with a 4-mm puncher (for the inner hole) and then with a $6.5-\mathrm{mm}$ puncher (for the outer hole). PMMA hollow cylinder ( $5 \mathrm{~mm}$ high, $1 \mathrm{~mm}$ thick) was made up with micromilling machine. The PDMS biochip was sterilized by autoclave, while PDMS gasket and PMMA cylinder were sterilized by UV light. Before the experiments, InLiverbiochip was washed twice with PBS and then was filled with cell culture medium without encapsulating air bubbles. Then, the 3DHIM was inserted in the transwell insert of the Intestine $e_{c}$, the PDMS gasket was placed on the surface of the 3D-HIM, and the PMMA cylinder was placed on the PDMS gasket. In addition, to avoid bacterial contaminations, the Intestine ${ }_{c}$ was closed by laying a $100-\mu \mathrm{m}$ sterile PDMS sheet fabricated by spin coating of PDMS $(750 \mathrm{rpm} \times 30 \mathrm{~s})$ onto transwell insert of the Intestine ${ }_{c}$. The entire set-up was connected to a syringe pump that worked at flow rate of $5 \mu \mathrm{L} / \mathrm{min}$, a reservoir was connected to the basal Intestine $_{c}$, and another one was connected to the In-Liver outlet in order to collect the cell supernatants from each compartment. For each experiment, two parallel devices were used. To simulate oral ingestion and toxic damage to both Intestine ${ }_{c}$ and $\operatorname{Liver}_{c}$, Et-OH dissolved into $300 \mu \mathrm{L}$ of culture medium $(400 \mathrm{mM})$ was added on the apical side of the Intestine ${ }_{c}$. The InLiver-OCs were incubated for $24 \mathrm{~h}$ at $37^{\circ} \mathrm{C}$ in a humidified atmosphere containing $5 \% \mathrm{CO}_{2}$. The control group InLiver-OC without Et$\mathrm{OH}$ treatment was named "-Et-OH;" the 400-mM Et-OH treated sample was named "+Et-OH." 3D-HIMs and HepG2- $\mu$ TPs were collected and processed for immune-histotypical and molecular characterizations. In addition, the media collected were analyzed for metabolites produced on the apical side of the Intestine ${ }_{c}$, on the lower side of the Intestine $e_{c}$ as well as on the outlet of the InLiver-OC.

\section{Mathematical Model CFD Simulation}

The commercial CFD COMSOL Multiphysics vers. Five was used to verify the experimental setup, the three-dimensional velocity and the oxygen gradients in the InLiver-OC. In the CDF analysis, 
TABLE 1 | Mathematical modeling variables.

\begin{tabular}{|c|c|}
\hline \multicolumn{2}{|l|}{ 3D HIM properties } \\
\hline Equilibrium $\mathrm{O}_{2}$ concentration $\left[\mathrm{C}_{0}\right]$ & $0.22 \mathrm{~mol} / \mathrm{m}^{3}$ \\
\hline 3D HIM Cell density $[\rho]$ & $0.2 \times 10^{14} \mathrm{cell} / \mathrm{m}^{3}$ \\
\hline Diffusion coefficient $\left(\mathrm{D}_{t}\right)$ & $10^{-10} \mathrm{~m}^{2} / \mathrm{s}$ \\
\hline Dynamic permeability [ $\left.k_{d v}\right]$ & $0 \mathrm{~m}^{2}$ \\
\hline Porosity of the tissue $[\varepsilon]$ & 0.7 \\
\hline Effective viscosity in the fluid $\left[\mu_{t}\right]$ & $0.0016 \mathrm{Pas}$ \\
\hline Effective hydraulic conductivity $\left[\mathrm{K}_{\mathrm{t}}\right]$ & $10^{-11} \mathrm{~m}^{2}$ \\
\hline Maximum oxygen consumption rate $\left(\mathrm{V}_{\max }\right)$ & $10^{-18} \mathrm{~mol} / \mathrm{cells}^{*} \mathrm{~s}$ \\
\hline $\mathrm{O}_{2}$ concentration at $V_{\max } / 2\left(K_{m}\right)$ & $10^{-3} \mathrm{~mol} / \mathrm{m} 3$ \\
\hline \multicolumn{2}{|l|}{ HepG2- $\mu$ TPs properties } \\
\hline Effective hydraulic conductivity $\left[\mathrm{k}_{\mathrm{t}}\right]$ & $10^{-11} \mathrm{~m}^{2}$ \\
\hline Porosity of the tissue $[\varepsilon]$ & 0.7 \\
\hline Dynamic permeability [k $\left.k_{d v}\right]$ & $0 \mathrm{~m}^{2}$ \\
\hline Diffusion coefficient $\left(D_{t}\right)$ & $10^{-10} \mathrm{~m}^{2} / \mathrm{s}$ \\
\hline Effective viscosity in the fluid $\left[\mu_{t}\right]$ & $0.0016 \mathrm{Pas}$ \\
\hline HepG2- $\mu$ TPs cell density $[\rho]$ & $2.4 \times 10^{13} \mathrm{cell} / \mathrm{m}^{3}$ \\
\hline Maximum oxygen consumption rate (Vmax) & $2.77 \times 10^{-17} \mathrm{~mol} / \mathrm{cells}^{*} \mathrm{~s}$ \\
\hline $\mathrm{O}_{2}$ concentration at $V_{\max } / 2\left(\mathrm{~K}_{m}\right)$ & $6.3 \times 10^{-3} \mathrm{~mol} / \mathrm{m}^{3}$ \\
\hline \multicolumn{2}{|l|}{ Culture media properties } \\
\hline Oxygen diffusivity $\left[D_{f}\right]$ & $10-9 \mathrm{~m}^{2} / \mathrm{s}$ \\
\hline Dynamic viscosity $\left[\mu_{\mathrm{f}}\right]$ & $0.001 \mathrm{~Pa} \mathrm{~s}$ \\
\hline
\end{tabular}

the entire InLiver-OC bioreactor was divided into two different parts, a fluid domain, indicated by " $\mathrm{f}$ " (culture medium), and a tissue domain, indicated by " $\mathrm{t}$ " (tissue equivalents). The oxygen transport and the three-dimensional velocity were evaluated by combining different physics. Fluid flow in tissue-free regions was modeled by using steady state Navier-Stokes Equation (1); fluid flow in tissue domains was modeled by using transport in porous media Equation (2); oxygen transport was modeled by using Mass Transport-Convection/Diffusion application mode Equation (3); the generation term for oxygen was modeled by using MichelisMenten kinetic Equation (4). Reference pressure was considered at device outlet $(p=0 \mathrm{~Pa})$; no slip condition was adopted at the walls; up to the intestine chamber a fixed concentration of $\mathrm{O}_{2}$ was imposed to simulate an open chamber; equality for velocity and pressure was imposed at the Navier-Stokes (u, v, w, p)/Brinkman $\left(\mathrm{u}_{2}, \mathrm{v}_{2}, \mathrm{w}_{2}, \mathrm{p}_{2}\right)$ interfaces. All values utilized in the simulation are reported in Table 1. Laminar flow with different flow rates was set at the inlet, and zero pressure was set at the outlet.

$$
\mu_{f} \nabla^{2} v_{f}=\nabla P_{f}
$$

Where $\mu_{f}$ is the dynamic viscosity, $\nu_{f}$ is the fluid velocity, and $P_{f}$ is the pressure. Brinkman Equation (2) was used to describe the flow through the porous medium:

$$
\mu_{t} \nabla^{2} v_{t}-K_{t} \mu_{t}=\nabla P_{t}
$$

Where $K_{t}$ is the hydraulic permeability, $\mu_{t}$ is the viscosity of the tissue, and $P_{t}$ is the pressure.
The oxygen concentration within the system was calculated using the following mass balance Equation (3):

$$
D \nabla^{2} C-\nabla(C v)=-R
$$

Where $C$ is the oxygen concentration, $v$ is the fluid velocity field that was set equal to $v f$ in the domain " $\mathrm{f}$ " and $v_{t}$ in the domain "t," respectively. $D$ is the diffusion coefficient of the oxygen, set as $D_{f}$ in the domain " $\mathrm{f}$ " and $\mathrm{D}_{\mathrm{t}}$ in the domain " $\mathrm{t}$ " respectively. $R$ is the volumetric oxygen consumption rate expressed by the Michaelis-Menten law, according to the following Equation (4):

$$
R=\frac{\rho V_{\max } C}{K_{m}+C}
$$

where $V_{\text {max }}$ is the maximum oxygen consumption rate, and $K_{m}$ is the concentration at which the oxygen consumption rate is half of $V_{\max }, \rho$ is the cell density in the perfusion chamber. For each tissue, corresponding values were considered. The HepG2 setting values were established from Weise et al. (2013). R was set to 0 only in the fluid domain, since cells are present only in the tissue domain.

\section{Culture Media Selection for Co-culture in InLiver-OC}

Two culture media (DMEM and EMEM) and different supplements were used for 3D-HIMs and HepG2- $\mu$ TPs, respectively. In order to determine the optimal medium composition to flow into InLiver-OC, different media were tested such as: In-medium (Enriched_DMEM), Liver-medium (Enriched_EMEM), and their combination at the following ratio $1: 1,1: 2$, and 2:1 ratio (v/v). MTT and LDH assays were used to establish the medium condition that guarantees the highest cell viability and lowest toxicity.

\section{Et-OH Curve and Cell Viability Assessment on 2D vs. 3D Liver Models}

In order to evaluate the cell viability after Et-OH treatment in $2 \mathrm{D}$ as well as in HepG2- $\mu$ TPs culture, 3-(4,5-dimethylthiazol2-yl)-2,5-diphenyltetrazolium bromide (MTT) and Lactate dehydrogenase (LDH) assays were used according to the manufacturer's instructions (Dojindo Molecular Technologies Inc., Rockville, MD). For MTT assay 2D cell cultures (HepG2 cells) were seeded at $5 \times 10^{3}$ cells for each well in 96-well cell culture plate and cultured for 3 days prior to the start of the experiments. Based on the generation time of the HepG2, the total number of the cells at the beginning of the MTT test was $\sim 2.52 \times 10^{4}$. For $3 \mathrm{D}$ culture, HepG $2-\mu$ TPs $(\sim 15$ microtissues, $2.32 \times 10^{4}$ total cells) were collected from the spinner flask at culture day 6 and loaded in 96-well cell culture plate. All samples were starved in culture medium with $0.2 \% \mathrm{FBS}$ overnight and then, the medium with different Et-OH concentrations (0, $100,200,300,400,500$, and $600 \mathrm{mM}$ ) was administered to $2 \mathrm{D}$ HepG2 and 3D HepG2- $\mu$ TPs and were cultured for $24 \mathrm{~h}$. For MTT assay, the optical density of each well sample was measured with a microplate spectrophotometer reader at $550 \mathrm{~nm}$. Then, to evaluate the $\mathrm{LDH}$ release, the culture supernatants from 
2D and 3D HepG2-Microtissues LDH activity was performed, using LDH Detection Kit (Sigma-Aldrich) according to the manufacturer's protocol. Briefly, $50 \mu \mathrm{L}$ of sample or $\mathrm{NADH}$ standard were added to a 96-well. Then, reaction mix was added to each well, and after $2-5 \mathrm{~min}$, the absorbance at $450 \mathrm{~nm}$ was measured using a microplate reader; in this way a $T_{\text {initial }}$ was fixed. Subsequent measurements were performed once every $5 \mathrm{~min}$. The final measurement [(A450) final] was the penultimate reading or the value before the most active sample was near or exceeds the end of the standard curve. The time of the penultimate reading was $T_{\text {final }}$. The LDH activity was evaluated by the following Equation (5):

$$
L D H=\frac{B \times \text { Sample diluition factor }}{\text { Reaction time }} \times V
$$

Where B is the amount (nmole) of NADH generated between $T_{\text {initial }}$ and $T_{\text {final }}$, reaction time is the difference between $T_{\text {final }}$ and $T_{\text {initial }}$, and $V$ is the sample volume. ADH was expressed as $\mathrm{mUnit} / \mathrm{mL}$.

\section{Cytotoxicity Assessment of Intestine-Metabolized Et-OH on HepG2- $\mu$ TPs}

An indirect estimation of the undigested $\mathrm{Et}-\mathrm{OH}$ that crosses the 3D-HIM, and of the Et-OH metabolites produced by EtOHtreated, 3D-HIM were determined by collecting supernatants from apical (3D-HIM $\mathrm{H}_{\text {apical supernatants }}$ ) and basal side (3DHIM $_{\text {basal supernatants }}$ ) of 3D-HIM treated or not with $400 \mathrm{mM}$ Et-OH at $\mathrm{T}_{0}$ or at $24 \mathrm{~h}$. MTT was performed on HepG2$\mu$ TPs treated with the 3D-HIM supernatants. In addition, zone of Inhibition Test on L. rhamnosus were performed after the treatment of the bacteria with the 3D-HIM supernatants. For Zone of Inhibition assay, transparent zones of bacterial grown inhibition indicated the antibacterial activity of the Et-OH. Image J software was used for the measurement of the diameter of the inhibition zones.

\section{Cytotoxic and Immunohistotypical Analyses of 3D-HIM Cultured in InLiver-OC} $3 \mathrm{D}$-HIMs cultured in InLiver-OC were either treated or not treated with $400 \mathrm{mM}$ Et-OH for $24 \mathrm{~h}$, then supernatants were collected, and LDH was performed as reported in section Et-OH Curve and Cell Viability Assessment on 2D vs. 3D Liver Models. Moreover, 3D-HIMs (control groups and Et-OH-treated) were fixed in a solution of $10 \%$ neutral buffered formalin for $1 \mathrm{~h}$, rinsed in PBS, dehydrated in an incremental series of alcohol $(75,85,95$, and $100 \%$ twice, each step $30 \mathrm{~min}$ at RT), then treated with xylene (30 min twice) and embedded in paraffin. Tissue sections of $5 \mu \mathrm{m}$ thick were stained with Hematoxylin and Eosin (H\&E) or Alcian blue, mounted with Histomount mounting solution (Bioptica) on coverslips, and the morphological features of 3D-HIMs were observed with a light microscope. To quantify the mucus secretion, samples were processed by using color deconvolution plugin (Image $\mathrm{J}^{\circledR}$ ). Automatic thresholding was applied, and blue staining (indicating mucus secretion) was separated from other colors. Further, the mucus deposition was quantified as the mean percentage of blue-stained region. Ten sections were used, and at least five different fields were randomly examined in each section for each time point. For immunofluorescence assay, 3D-HIMs were withdrawn from the InLiver-OC and fixed with $4 \%$ paraformaldehyde for $20 \mathrm{~min}$ and then rinsed with PBS. The samples were then incubated with a permeabilization solution $(0.2 \%$ Triton X-100 + 3\% BSA + PBS $)$ for 10 min. After blocked for $1 \mathrm{~h}$ at RT, primary antibody (Claudin-1, 1/40, Abcam Laminin V, 1/50, Abcam; MMP-9, 1/250, Abcam) was incubated for $1 \mathrm{~h}$ at RT. Then, secondary antibody incubation, donkey AlexaFluor 546-conjugated anti-rabbit IgG antibodies, for Claudin-1 and Laminin $\mathrm{V}$ and goat AlexaFluor 546-conjugated anti-mouse for MMP-9 were incubated for $1 \mathrm{~h}$. Cells nuclei were detected by DRAQ5 staining (5 $\mathrm{m} / \mathrm{mL}$, Sigma Aldrich).

\section{Quantification of Tight Junction Formation}

For epithelial barrier evaluation, the 3D-HIMs were withdrawn from the InLiver-OC, and the quality of tight junctions was assessed by means of Claudin-1 staining. Cell samples were immune-stained for Claudin-1 and examined with Leica (Confocal Leica TCS SP5 II femtosecond laser scanning system, Leica) confocal microscope. For each sample, the number of tight junction structures, defined as a completely enclosed ring of smooth, contiguous, apical Claudin-1 staining, were counted in three different fields under $20 \mathrm{X}$ magnification ( $>50$ cells per field), and the mean number of tight junction structures per field was determined. Experiments were performed three times, and the mean number of tight junction structures per field from three independent experiments was calculated.

\section{Transepithelial Electrical Resistance}

The transepithelial electrical resistance (TEER) of the 3D-HIM cultured in InLiver-OC was measured by using an Autolab PGSTAT101 (potentiostat/galvanostat, Metrohm) equipped with FRA32M module (frequency response analysis module), which allowed for obtaining values of electrical impedance on a proper range of frequencies $(10 \mathrm{~Hz}-10 \mathrm{kHz})$. Measurements were carried out using the two electrodes setup in potentiostatic mode, with an amplitude of $0.3 \mathrm{~V}$ _RMS and an AC current range of $10 \mu \mathrm{A}$ (to avoid damage of the 3D-HIM); two platinum electrodes $(0.38 \mathrm{~mm}$ diameter and $99.9 \%$ purity) were used. In order to obtain TEER values, impedance curve (40 data, 10 points per decade) was fitted with non-linear least-square method using software Nova2.1. In particular, the adopted model consisted of a series of a resistor (resistance of blank solution), CPE element (interaction between solution and electrodes), and a parallel between a resistor (TEER) and a capacitor (cell membrane) (Figure S2).

\section{ECM Microarchitecture of 3D-HIM}

Two-photon microscopy (Leica TCS SP5 II coupled with a multiphoton microscope where the NIR femtosecond laser beam was derived from a tunable compact mode locked titanium: sapphire laser-Chamaleon Compact OPO-Vis, Coherent) was used to produce Second Harmonic Generation (SHG) images in which unstained collagen in 3D-HIMs (untreated or Et-OHtreated) were highlighted. We used an excitation wavelength of 
$\lambda_{\text {ex }}=840 \mathrm{~nm}$ (two photon) and collected the signal at emission wavelength in the range $\lambda_{\text {em }}=420 \pm 5 \mathrm{~nm}$. ImageJ was used for the quantification of the collagen fraction (CF) by measuring the collagen portion in the ECM space in a selected region of interest (ROI). The collagen portion in the ECM corresponds to bright pixels with respect to black pixels, which represent the non-collagen portion. The CF was expressed as the ratio of bright pixels to total pixels (bright pixels + black pixels) in terms of percent in the selected ROI. In addition, the collagen assembly degree (CAD) was evaluated by analyzing the intensity of the SHG signal. All SHG images were subjected to noise subtraction, and the average intensity was evaluated as described by Equation (6):

$$
C A D \propto \overline{\mathrm{I}}=\frac{\sum_{i=1}^{255}\left(I_{i} p_{i}\right)}{\sum_{i=1}^{255}\left(p_{i}\right)}
$$

where $\overline{\mathrm{I}}$ is the average intensity, $I_{i}$ is the intensity corresponding to the pixel, $p_{i}$, while the index, $i=\mathrm{x}_{\mathrm{i}}, \mathrm{y}_{\mathrm{i}}$, runs in the gray value interval from 1 to 255 . The intensity $\overline{\mathrm{I}}$ of the collagen network is known to be proportional to the degree of assembly of the newly synthesized collagen (De Gregorio et al., 2019). To quantify the stroma related changes, we performed Graylevel-Co-occurrence Matrix (GLCM) texture analysis, by using the Image J plug-in "Texture" on SHG images. In this work, we calculated the correlation curve (COR) for distances ranging from 1 to 100 pixels in the horizontal $\left(0^{\circ}\right)$ and vertical $\left(90^{\circ}\right)$ direction of each optical section that cover a length of interest of $40 \mu \mathrm{m}$. In such spatial windows, the distance at which the correlation function falls off represents the correlation length of the texture. In particular, correlation curve was calculated vs. neighbor index, and correlation length was obtained by fitting data with an exponential law. The Equation (7) of COR is given, below:

$$
\mathrm{COR}=\sum_{i, j} \frac{\left(i-\mu_{i}\right)\left(j-\mu_{j}\right) p(i, j)}{\sigma_{i} \sigma_{j}}
$$

where $\mu_{i}, \mu_{j}, \sigma_{i}$, and $\sigma_{j}$ are given by Equations (3-6):

$$
\begin{aligned}
\mu_{i} & =\sum i, j i * p(i, j) \\
\mu_{j} & =\sum i, j j * p(i, j) \\
\sigma_{i} & =\sqrt{\sum i, j\left(1-\mu_{i}\right)^{2} p(i, j)} \\
\sigma_{j} & =\sqrt{\sum i, j\left(1-\mu_{j}\right)^{2} p(i, j)}
\end{aligned}
$$

In particular $p(i, j)$ is the probability of gray level $i$ occurring next to gray level $j, \mu_{i}, \mu_{j}, \sigma_{i}$, and $\sigma_{j}$ are the means $\mu$ and standard deviations $\sigma$ of column $i$ and line $j$ of the matrix, respectively. All parameters have a maximum value of 1 and a minimum value of 0 or -1 . The COR curve is an index of the architecture of the network, with a fast decay for fine textures and slow decay for coarse structure. From the COR curve, it is possible to obtain the correlation length, $\lambda$, defined as the distance at which the COR decay is equal to 0.5 .

\section{Morphological, Functional and Immunohistotypical Analyses on HepG2- $\mu$ TPs Cultured in Liver-OC or InLiver-OC}

HepG2- $\mu$ TPs (control groups and Et-OH-treated) withdrawn from the Liver-OC or InLiver-OC were processed for histological and immunofluorescence analyses, as reported in section Cytotoxic and Immunohistotypical Analyses of 3D-HIM Cultured in InLiver-OC. For immunofluorescence assay, primary antibody (Claudin-1, 1/40, Abcam; P-gp, 1/50, Abcam) and secondary antibody (donkey AlexaFluor 546-conjugated anti-rabbit IgG antibodies for Claudin-1, goat Alexa Fluor 488-conjugated anti-mouse IgG antibodies for P-gp) were used. Cells nuclei were detected by DRAQ5 staining $(5 \mu \mathrm{m} / \mathrm{mL}$, Sigma Aldrich). Furthermore, in order to measure albumin and urea released by HepG2- $\mu$ TPs, culture media were collected after Et-OH administration on HepG2- $\mu$ TPs cultured in Liver-OC. Specifically, the supernatants were centrifuged at 2,000 g for $10 \mathrm{~min}$ in order to remove cell debris. They were then stored at $-20^{\circ} \mathrm{C}$ before being analyzed. Albumin secretion in cell culture supernatants was quantified by a sandwich enzyme-linked immunosorbent assay (ELISA) kit (Abcam), according to the manufacturer's instructions. All experiments were performed in triplicate. Urea levels in cell supernatants were determined by Quanti Chrom TM Urea Assay Kit (DIUR-500) according to the manufacturer's instruction.

\section{Oil Red Staining on HepG2- $\mu$ TPs}

In order to evaluate the lipids production and localization in HepG2- $\mu$ TPs within InLiver- and Liver-OC treated or not treated with $400 \mathrm{mM}$ Et-OH for $24 \mathrm{~h}$, oil red staining was performed. A group of samples was stained directly into the device, and another group was fixed in formalin 10\%. The paraffin embedded samples were cut into $4-\mu \mathrm{m}$-thick sections, which were mounted on coating slides, unmasked with antigen retrieval and stained with oil red (Sigma Aldrich). Briefly all the samples were incubated for $5 \mathrm{~min}$ with $60 \%$ isopropanol at room temperature, rinsed in $\mathrm{dH}_{2} \mathrm{O}$, and stained for 30 min with oil red working solution. Then, the samples were counterstained with hematoxylin, washed thoroughly with $\mathrm{dH}_{2} \mathrm{O}$, and transferred to mounting medium (Bio Optica).

\section{Reactive Oxygen Species (ROS) Production on HepG2- $\mu$ TPs}

HepG2- $\mu$ TPs were collected from InLiver- or Liver-OC with or without $400 \mathrm{mM}$ Et-OH treatment for $24 \mathrm{~h}$, in order to assess Reactive Oxygen Species (ROS) production. After the incubation, all samples were washed with PBS and incubated with $10 \mu \mathrm{M}$ CM-DCFDA (5-6)-chloromethyl2,7 dichlorodihydrofluoresceine diacetate (ROS indicator, Invitrogen) at $37^{\circ} \mathrm{C}$ for $60 \mathrm{~min}$ in dark condition. For positive control groups, the samples were incubated with $\mathrm{H}_{2} \mathrm{O}_{2} 400 \mu \mathrm{M}$ 
for $30 \mathrm{~min}$. Then, all samples were washed in PBS, returned in pre-warmed growth medium, and visualized under confocal microscope (Confocal Leica TCS SP5 II femtosecond laser scanning system, Leica), using excitation appropriate source for fluorescein (FITC). For quantitative analysis, 20 images were processed by using ImageJ software, and the green signal (ROS indicator excitation at $485 \mathrm{~nm}$ ) was normalized to the surface area.

\section{Metabolic and Molecular Assay on 3D-HIMs and HepG2- $\mu$ TPs Cultured in InLiver-OC}

\section{Alcohol Dehydrogenase Release Assay}

In order to evaluate the alcohol dehydrogenase $(\mathrm{ADH})$ release, the culture supernatants from In-Liver-OC treated or not treated with $400 \mathrm{mM}$ Et-OH for $24 \mathrm{~h}$ were collected and stored at $-20^{\circ} \mathrm{C}$ before being analyzed. $\mathrm{ADH}$ activity was performed, using Alcohol Dehydrogenase Activity Detection Kit (Abcam), according to the manufacturer's protocol. Briefly, $50 \mu \mathrm{L}$ of sample or NADH standard were added to a 96-well. Then reaction mix was added to each well, and the absorbance at $450 \mathrm{~nm}$ was measured using a microplate reader after 2-5 min; in this way, a $T_{\text {initial }}$ was fixed. Subsequent measurement was performed after $2 \mathrm{~h}$. The $\mathrm{ADH}$ activity was evaluated by the following Equation (12):

$$
A D H=\frac{B \times \text { Sample diluition factor }}{\text { Reaction time }} \times V
$$

where $B$ is the amount (nmole) of $\mathrm{NADH}$ generated between $T_{\text {initial }}$ and $T_{\text {final }}$, reaction time is the difference between $T_{\text {final }}$ and $T_{\text {initial }}$, and $V$ is the sample volume. ADH was expressed as $\mathrm{mUnit} / \mathrm{mL}$. All experiments were performed in triplicate.

\section{Reverse Transcription-Polymerase Chain Reaction Analysis}

FFPE tissues blocks (3D-HIMs and HepG2- $\mu$ TPs) was deparaffinized, digested, and total RNA was extracted by using RNeasy FFPE kit (Qiagen, Valentia, CA). Agarose gel electrophoresis assessed RNA integrity, and RNA concentrations were determined by UV light BioRad Imaging System (Biorad). $0.2 \mu \mathrm{g}$ of total cellular RNA were reverse-transcribed (Expand Reverse Transcriptase, Roche Diagnostics; Milan, Italy) into complementary DNA (cDNA) using random hexamer primers (Random hexamers, Roche Diagnostics; Milan, Italy), at $42^{\circ} \mathrm{C}$ for $45 \mathrm{~min}$. Two microliter of cDNA was amplified in a final volume of $50 \mu \mathrm{l}$ composed by $10 \mathrm{mM}$ Tris- $\mathrm{HCl}$ ( $\mathrm{pH} 8.3$ ), $1.5 \mathrm{mM} \mathrm{MgCl}_{2}$, $50 \mathrm{mM} \mathrm{KCl}, 200 \mu \mathrm{m} \mathrm{dNTP}$ and $2.5 \mathrm{U}$ of Taq DNA polymerase (Roche Diagnostics). The reaction was carried out in a DNA thermal cycler (Applied Biosystem). The expression of the following genes was examined: MRP, P-gp, and glyceraldehyde3-phosphate dehydrogenase (GAPDH) used as a housekeeping gene. The primers used for amplification were: MRP, $5^{\prime}$ tgatgagccgtatgtttgc $-3^{\prime}$ and $5^{\prime}$ - cttcggaacggacttgacat $-3^{\prime}$; P-gp, $5^{\prime}$ - atatcagcagcccacatcat $-3^{\prime}$ and $5^{\prime}$ - gaagcactgggatgtccggt $-3^{\prime}$; GAPDH, $5^{\prime}-\quad$ ccacccatggcaaattccatggca $-3^{\prime}$, and $5^{\prime}$ - tctagactggcaggtcaggtccacc-3'. PCR products were analyzed by electrophoresis on $1.8 \%$ agarose gel in TBE. Densitometric analysis of ethidium bromide-stained agarose gel was carried out using Image J analysis and normalized by the housekeeping gene GAPDH. Each data point represented the mean-standard error of the mean of three biological replicates.

\section{Solid Biomass Evaluation}

In order to determine the biomass weight of dehydrated samples, supernatants from In-OC, Liver-OC, or In-Liver-OC were collected and centrifuged at 13,000 rpm per $15 \mathrm{~min}$. All pellets were dehydrated in an incremental series of alcohol [30, 50, 75, 85,95 , and $100 \%$ twice], each step $30 \mathrm{~min}$ at RT, and then were made to evaporate under a chemical hood for $24 \mathrm{~h}$. After that, they were weighed on an analytical balance (Xs105 dual range, Mettler Toledo).

\section{Statistical Analyses}

Results were expressed as the mean \pm standard deviation (s.d.) from three or more independent experiments $(n \geq 3)$. For section staining, three specimens were used for each experiment and five sections were stained per specimen, then about five ROIs were examined for each section. All results were then statistically analyzed by the Student's $t$-test. The differences between two or more groups were evaluated using one-way analysis of variance (ANOVA) followed by the Tukey's post-test. Statistical significance was set at a value of $p<0.05$.

\section{RESULTS}

\section{Assessment of Intestinal and Epithelial Markers in 3D-HIM and HepG2- $\mu$ TPs}

Immunofluorescence staining was performed to verify the expression and localization of a representative intestinal epithelial marker, such as villin. As depicted in Figure 1a, the positivity for villin signal suggested the Caco-2 differentiation toward enterocytes cells showing highly polarized epithelial cells. In addition, the presence of microvilli structure on the free surface of the epithelial cells was also evidenced by ultrastructural SEM analysis, as showed in Figure 1a inset, confirming the epithelial polarization. The morphological features of the HepG2- $\mu$ TPs was investigated via immune-histochemical analysis. A detectable expression of the Cyt P450, a membraneassociated protein correlated with toxic compounds metabolism, was displayed (Figure 1b, *high magnification inset).

\section{InLiver-OC Assembly, Operation, and Comsol Simulation}

The designed InLiver-OC device allowed the co-culture of the two 3D organ models (3D-HIM in the Intestine ${ }_{c}$ and HepG2- $\mu$ TPs in the Liver $_{c}$ ) into separate compartments connected by means of microfluidic channel allowing an efficient unidirectional transport from the 3D-HIM to the HepG2$\mu$ TPs, mimicking the first-pass metabolism (Figures 2A,B). The Intestine $_{c}$ presents a central hole to accommodate a commercially available transwell insert in order to obtain two separate Intestine side (apical and basal). The $\operatorname{Liver}_{\mathrm{c}}$ was located downstream to 

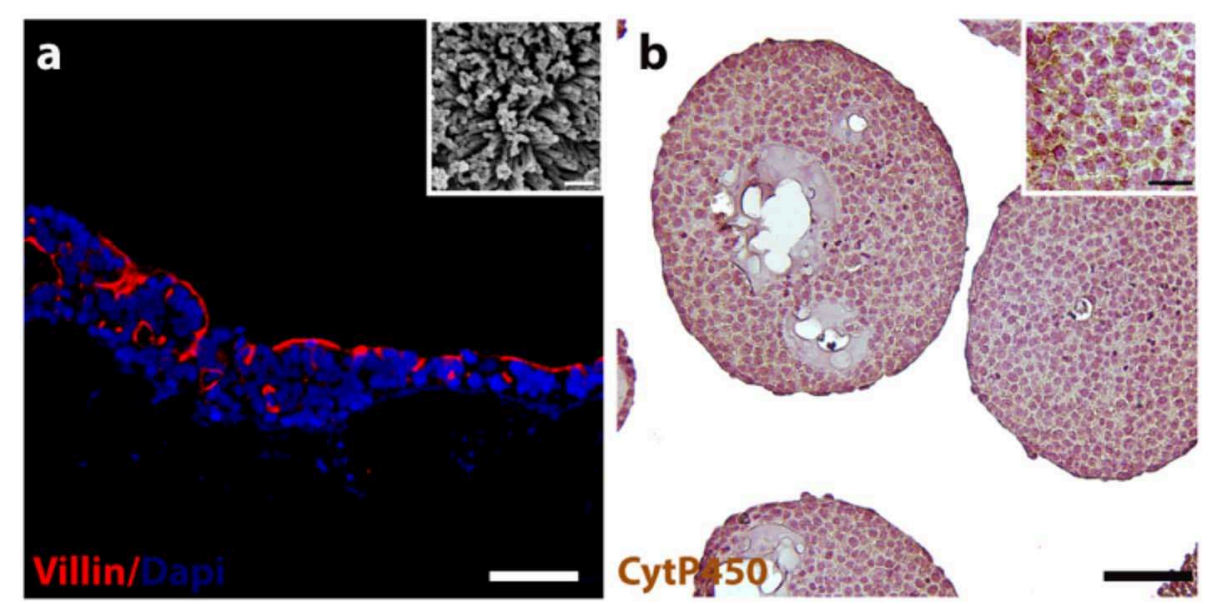

FIGURE 1 | 3D-HIM and HepG2- $\mu$ TPs functionality. Immunofluorescence analysis reveals the epithelial cells marker stained in red (villin) and nuclei in blue (DAPI) (a); scale bar, $75 \mu \mathrm{m}$. High magnification SEM micrograph shows the brush border on the apical side of the villi-like structures of the 3D-HIM (a, inset); scale bar 200 nm; representative immune-histochemical image of Cyt P450 on HepG2- $\mu$ TPs (b) and high magnification inset, scale bar 75 and $50 \mu m$, respectively.

the Intestine $e_{c}$ and was connected through a microchannel to the basal side of the Intestine ${ }_{c}$. 3D-HIM and HepG2- $\mu$ TPs were produced as previously reported (De Gregorio et al., 2018a; Corrado et al., 2019), and when the best performance in terms of viability and functionality (5 weeks for 3D-HIM and 5-7 days for HepG2- $\mu$ TPs) were detected, 3D-HIM was aseptically transferred into the apical side of the Intestine ${ }_{c}$, and HepG2$\mu$ TPs were loaded in each chamber of the $\operatorname{Liver}_{c}$ by pipetting. In order to assure the optimal conditions for cell survival, CFD (Figure 2) was used for simulating fluid dynamic conditions and oxygen concentration profile inside the InLiver-OC device. The flow rate at inlet $(5 \mu \mathrm{L} / \mathrm{min}$ ) assured the oxygen supply to both $3 \mathrm{D}$ models. As showed in Figure 2C, the $\mathrm{O}_{2}$ concentration in the $3 \mathrm{D}$-HIM was $0.16 \mathrm{~mol} / \mathrm{m}^{3}$, while the minimum $\mathrm{O}_{2}$ concentration in the HepG2- $\mu$ TPs was $0.12 \mathrm{~mol} / \mathrm{m}^{3}$. In Figure 2D, the velocity profile was shown, the maximum value of the fluid flow along the central channel was $5.4 \times 10^{-4} \mathrm{~m} / \mathrm{s}$ which is safe for cell viability according to literature (Sibilio et al., 2019).

\section{Selection of Medium Mixing for the Optimal 3D Co-culture in InLiver-OC}

Preliminary experiments were carried out in order to select the optimal media combination that did not cause the metabolic waste accumulation into the $\operatorname{Liver}_{\mathrm{c}}$. MTT and $\mathrm{LDH}$ assays (Figures 3A,B, respectively) were performed to establish the best medium combinations. We found that when 3D-HIM was cultured with the Liver-medium (Enriched_EMEM) or with InLiver-media (Enriched_DMEM:Enriched_EMEM at ratio $1: 2$ ), the viability of $3 \mathrm{D}-\mathrm{HIM}$ was marginally reduced (86 $\pm 7.12 \%$ and $88 \pm 6.22 \%$, respectively), and low value of $\mathrm{LDH}$ was detected $(0.2 \pm 0.04$ and $0.16 \pm 0.02$ munits $/ \mathrm{mL}$, respectively). At the same media combination, the HepG2$\mu$ TPs viability was slightly affected $(87 \pm 4.44 \%$ and $90.5 \pm$ $4.2 \%$, respectively), and a small but significant amount of $\mathrm{LDH}$ $(0.35 \pm 0.18$ and $0.21 \pm 0.07$ munits $/ \mathrm{mL}$, respectively) was detected, indicating that the 3D-HIM probably produced a small amount of metabolic waste that slightly induced the epithelial cell injury of the HepG2- $\mu$ TPs. In addition, when HepG2$\mu$ TPs were cultured with In-medium (Enriched_DMEM) or with InLiver-media (Enriched_DMEM:Enriched_EMEM at ratio $2: 1)$, a slight amount of $\mathrm{LDH}$ was revealed $(0.12 \pm 0.02$ and $0.11 \pm 0.04$ munits $/ \mathrm{mL}$, respectively), although these values were not indicative of cellular damage. Therefore, the optimal media combination resulted in the InLiver-media (Enriched_DMEM:Enriched_EMEM at ratio 1:1) that did not affect viability ( $96 \pm 8.3 \%$ for $3 \mathrm{D}$-HIM, $98 \pm 6.5 \%$ for HepG2$\mu \mathrm{TPs})$ and did not lead to the production of $\mathrm{LDH}(0.04 \pm 0.01$ for $3 \mathrm{D}$-HIM and $0.02 \pm 0.005$ munits $/ \mathrm{mL}$ for HepG2- $\mu$ TPs, respectively) as reported in Figures 3A,B, respectively.

\section{Selection of the Optimal Et-OH Concentration for Evaluating HepG2- $\mu$ TPs Damage}

Initial experiments were focused on understanding how the HepG-2 cells respond to different concentration of Et-OH. In this experimental phase, the cytotoxic effect of Et-OH was assessed on standard culture dishes (2D HepG-2 monolayer) or in 3D configuration (3D HepG2- $\mu$ TPs) (Figure 3C) by MTT viability assay. In detail, the 2D HepG-2 monolayer were seeded in 96 multiwell plate for $60 \mathrm{~h}$ in order to obtain $2.5 \times 10^{4}$ cells for each well. Instead, the 3D HepG2- $\mu$ TPs were cultured in a spinner flask in dynamic conditions in order to obtain 1,550 cell/ $\mu$ TPs; then on the sixth day, 5 HepG2- $\mu$ TPs were added for each liver chamber for a total of 15 HepG2- $\mu$ TPs $\left(2.32 \times 10^{4}\right.$ total number of cells), as shown in the Figure S3. For 2D HepG2 monolayer, a dose-dependent reduction of cell viability was reported from 0 to $600 \mathrm{mM}$ Et-OH. For 3D HepG2- $\mu$ TPs, a slight reduction of viability was revealed by adding $200 \mathrm{mM}$ Et-OH, but an increase in cell cytotoxicity was shown at concentration over $300 \mathrm{mM}$. Specifically, when 2D HepG-2 monolayer was 
A

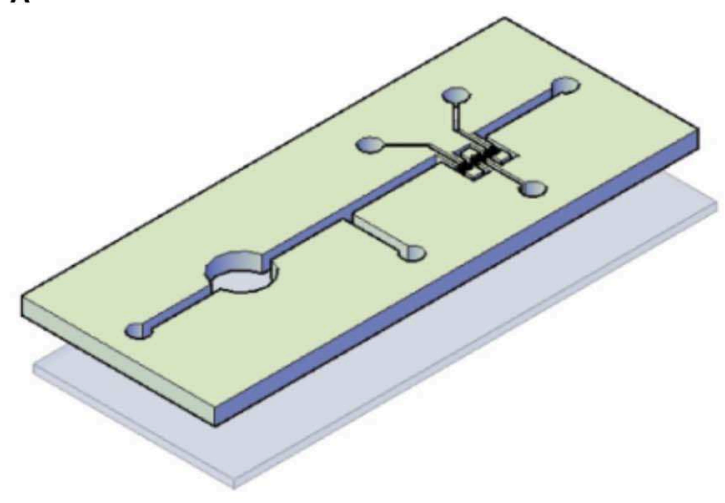

C

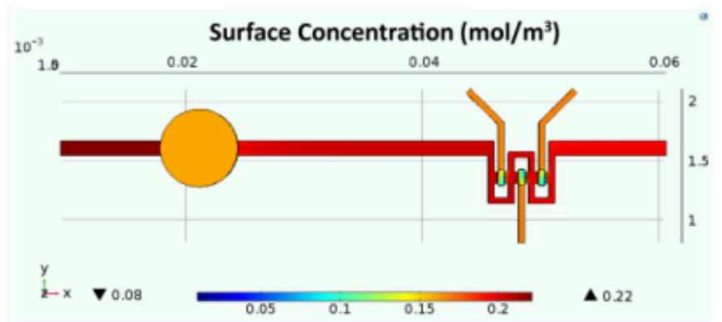

B

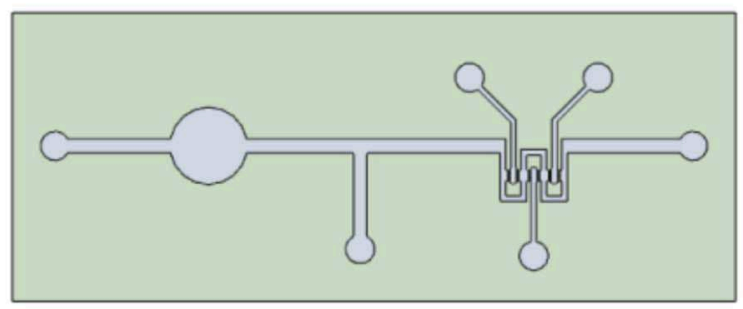

D

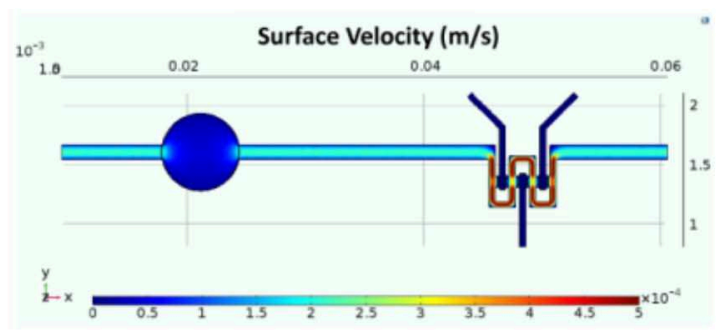

FIGURE 2 | Schematic representation of the InLiver-OC and CFD study. Lateral (A) and top (B) view of the InLiver biochip with Intestine ( $_{\mathrm{C}}\left(\mathrm{In}_{\mathrm{c}}\right)$ and Liver $\left(\right.$ aiv $\left._{\mathrm{c}}\right)$; fluid dynamic simulation indicates the velocity field in the device system at a flow rate of $5 \mu \mathrm{L} / \mathrm{min}$ (C) and the oxygen concentration field in the device working at a flow rate of $5 \mu \mathrm{L} / \mathrm{min}$ (D)

treated with Et-OH $400 \mathrm{mM}$, a significant reduction in cell vitality $(44 \pm 4.08 \%)$ compared to $3 \mathrm{D}$ HepG2- $\mu$ TPs treated with the same concentration $(61.9 \pm 5.02 \%)$ was revealed. In the groups treated with $500 \mathrm{mM}$ Et-OH, the viability percentage of the cells decreased at $32 \pm 4.3 \%$ in $2 \mathrm{D}$ HepG-2 monolayer and at 49.07 $\pm 5.32 \%$ in $3 \mathrm{D}$ HepG2- $\mu$ TPs. Finally, the cells treated with $600 \mathrm{mM}$ showed a mortality that exceeds $80 \%$ in $2 \mathrm{D}$ HepG-2 monolayer and was around $60 \%$ in $3 \mathrm{D} \mathrm{HepG} 2-\mu \mathrm{TPs}$. In order to perform the experiment in conditions that guarantee the cells functionality by preserving more than $50 \%$ of cell viability, the Et-OH concentration of $400 \mathrm{mM}$ was selected.

\section{0 mM Et-OH Effect on HepG2- $\mu$ TPs Metabolic Activity in Liver-OC}

In order to determine the liver functionality, the response of the HepG2- $\mu$ TPs to Et-OH treatment at $24 \mathrm{~h}$ was analyzed by measuring albumin and urea production. As shown in Figures 3E,F, the untreated samples (-Et-OH) exhibited high levels of albumin $(51.84 \pm 3.9 \mathrm{ng})$ and urea $(20.52 \pm 2.3$ $\mu \mathrm{g})$. In contrast, there was a significant decrease in the metabolic activity in $400 \mathrm{mM}$ Et-OH-treated samples (+Et-OH). In particular, albumin production decreased at value of 13.56 $\pm 3.81 \mathrm{ng}$ and urea at $8.39 \pm 1.48 \mu \mathrm{g}$, demonstrating that the HepG2- $\mu$ TPs responded to the toxic stimulus by reducing their metabolic activity.

\section{Intestine-Metabolized Et-OH Affects HepG2- $\mu$ TPs Viability and $L$. rhamnosus Growth}

An indirect estimation of the undigested Et-OH crossing the 3DHIM was evaluated by treating HepG2- $\mu$ TPs and L. rhanmosus with tissue supernatants in order to perform cell viability assay (MTT) and Zone of Inhibition Test, respectively (Figures 4A,B). Specifically, HepG2- $\mu$ TPs were treated with the 3D-HIM supernatants collected from apical $\left(3 \mathrm{D}-\mathrm{HIM}_{\text {apical supernatants }}\right)$ or basal side $\left(3 \mathrm{D}-\mathrm{HIM}_{\text {basal supernatants }}\right.$ ) of the Intestine $\mathrm{c}_{\mathrm{c}}$ at $\mathrm{T}_{0}$ or after $24 \mathrm{~h}$ of $400 \mathrm{mM}$ Et-OH treatment. The results indicated that at $\mathrm{T}_{0}$ the $3 \mathrm{D}$-HIM $\mathrm{H}_{\text {apical supernatants }}$ slightly reduced HepG2$\mu$ TPs viability, resulting in cell vitality of $62.5 \pm 5.2 \%$. This value corresponded in the Et-OH dose response curve to an Et-OH concentration of $400 \mathrm{mM}$ (Figure S4). No significant reduction in cell viability was induced by treating HepG2- $\mu$ TPs with 3DHIM $_{\text {basal supernatants }}$ harvested at $\mathrm{T}_{0}$. HepG2- $\mu$ TPs treated with $3 \mathrm{D}$-HIM $\mathrm{H}_{\text {apical supernatants }}$ collected at $24 \mathrm{~h}$, revealed a very slight reduction of cell viability ( $87 \pm 5.4 \%$ cell viability), while HepG2$\mu$ TPs treated with $3 \mathrm{D}-\mathrm{HIM}_{\text {basal supernatants }}$ harvested after $24 \mathrm{~h}$ of Et-OH treatment, presented a more pronounced reduction in cell viability ( $74 \pm 5.3 \%$ cell viability). The latter value corresponded in the dose response curve (Figure 3D) to an Et$\mathrm{OH}$ concentration of $350 \mathrm{mM}$, suggesting that a small amount of Et-OH was metabolized by the 3D-HIM (Figure 4A) at $24 \mathrm{~h}$. 

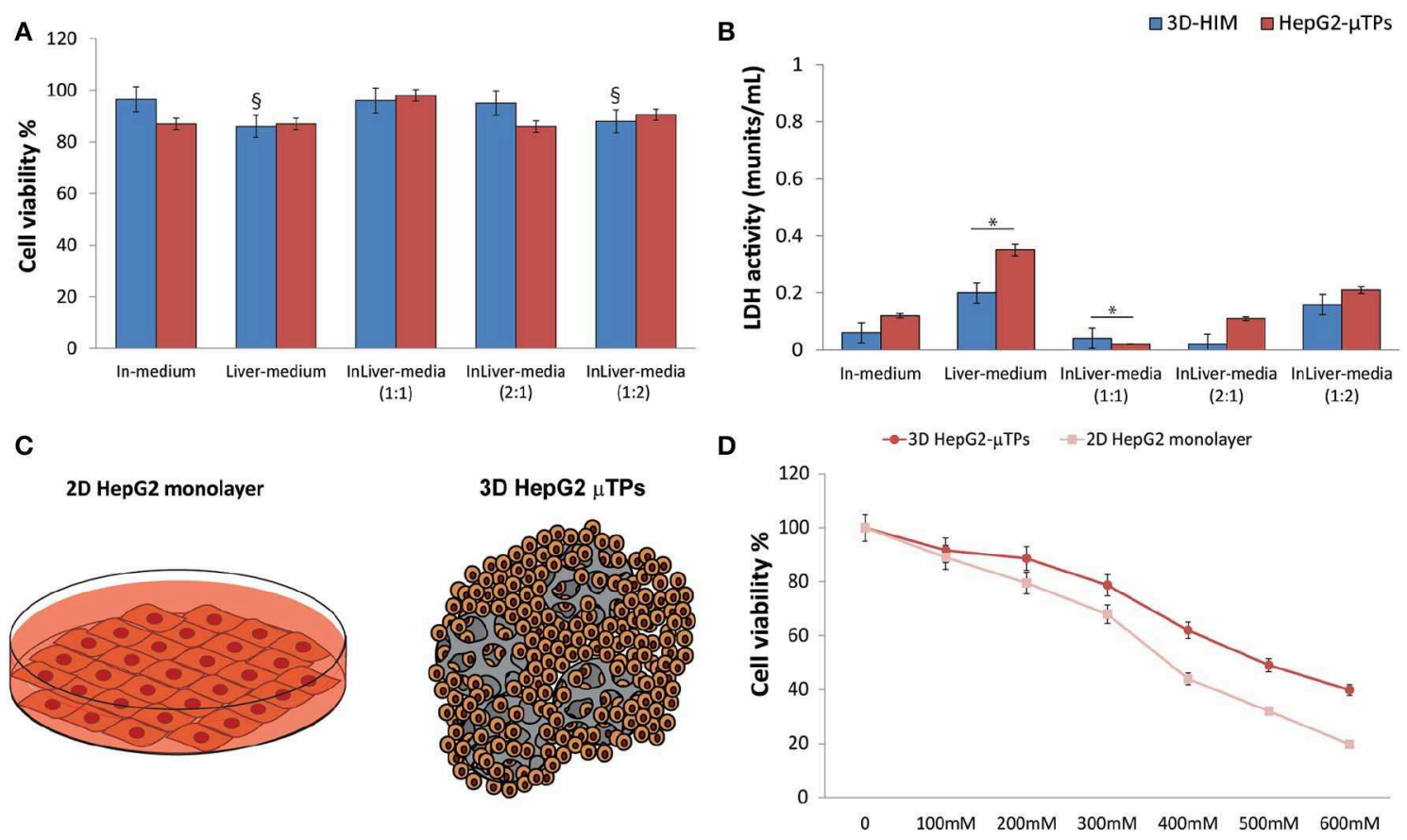

E

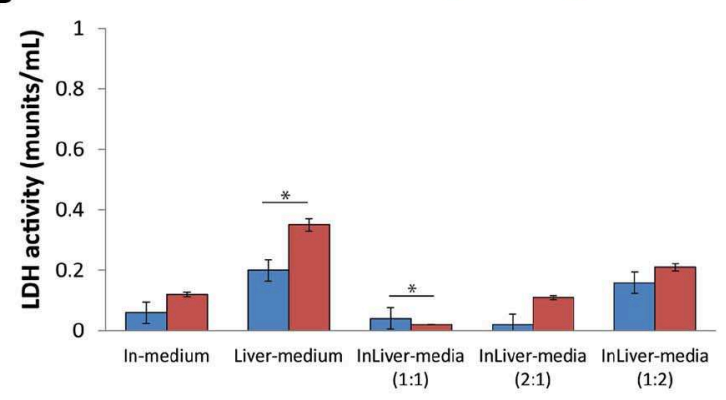

$\mathbf{F}$
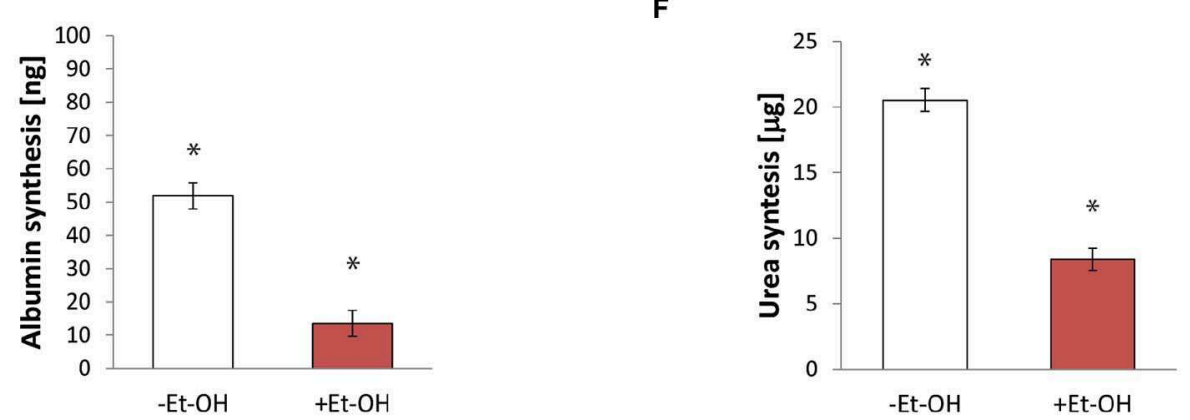

FIGURE 3 | Selection of optimal media combination in InLiver-OC, HepG-2 cytotoxicity under different Et-OH concentrations and 3D HepG2- $\mu$ TPs metabolic activity under $400 \mathrm{mM}$ Et-OH. MTT cell viability (A) and LDH (B) assays on 3D-HIM and HepG2- $\mu$ TPs cultured with different media combinations $\left({ }^{\S} p<0.05 ;{ }^{*} p<0.05\right)$; schematic representation of standard culture dishes (2D HepG2 monolayer) and 3D configuration (3D HepG2- $\mu$ TPs) (C); dose response on 2D HepG2 monolayer vs. 3D HepG2- $\mu$ TPs at different Et-OH concentrations (0-600 mM) (D); comparison of Albumin (E) and urea production (F) on untreated (-Et-OH) and $400 \mathrm{mM}$

Et-OH-treated (+Et-OH) 3D HepG2- $\mu$ TPs. The Albumin amount is expressed in "ng" and the urea synthesis is indicated in " $\mu$ g." All the experiments were performed in triplicate $(n=3)$, Values represent the mean and the standard deviation $\left({ }^{*} p<0.05\right)$.

Further, the antibacterial action of Et-OH was evaluated by analyzing the supernatants collected from $3 \mathrm{D}-\mathrm{HIM}_{\text {apical }}$ treated or not treated with $400 \mathrm{mM} \mathrm{Et-OH}$ at $\mathrm{T}_{0}$ and $24 \mathrm{~h}$. The maximum zone of L. rhamnosus strain inhibition growth $(2.1$ $\pm 0.51 \mathrm{~cm})$ was found by using supernatants collected at $\mathrm{T}_{0}$ from Et-OH treated 3D-HIM apical while a relatively lower $(0.8 \pm$ $0.66 \mathrm{~cm}$ ) growth inhibition zone was found by using supernatants collected from Et-OH treated $3 \mathrm{D}-\mathrm{HIM}_{\text {apical }}$ at $24 \mathrm{~h}$. Moreover, there was no antibacterial effect of the $3 \mathrm{D}-\mathrm{HIM}_{\text {basal supernatants }}$ at $\mathrm{T}_{0}$, but an inhibition of the growth zone $(1.5 \pm 0.5 \mathrm{~cm})$ was observed when $3 \mathrm{D}-\mathrm{HIM}_{\text {basal supernatants }}$ at $24 \mathrm{~h}$ was used. Taken together, these results demonstrate that Et-OH or Et-OH products such as acetaldehyde reduce the growth of L. rhamnosus strain (Figure 4B).

\section{Et-OH-Induced 3D-HIM Hyper-Permeability and Stromal Injury in InLiver-OC}

In order to evaluate the harmful effect of $400 \mathrm{mM}$ Et-OH on 3D-HIM, the cell damage was assessed by LDH assay. The Et-OH was given on the apical side of the 3D-HIM, and at this dose, an LDH release of $0.92 \pm 0.07 \mathrm{mU} / \mathrm{mL}$ at $24 \mathrm{~h}$ was found (Figure 4C), suggesting slight Et-OH-induced cell damage. This Et-OH concentration also impacted 3D-HIM's morphology and functional performance. In order to evaluate the effect in terms of the intestinal barrier integrity, intestinal tight junctions were investigated. High intensity of Claudin-1 signal, a tight junction marker, was observed on untreated samples (-Et-OH) compared to the Et-OH-treated ones (+Et-OH), in which the higher amount of acetaldehyde compromises the tight 

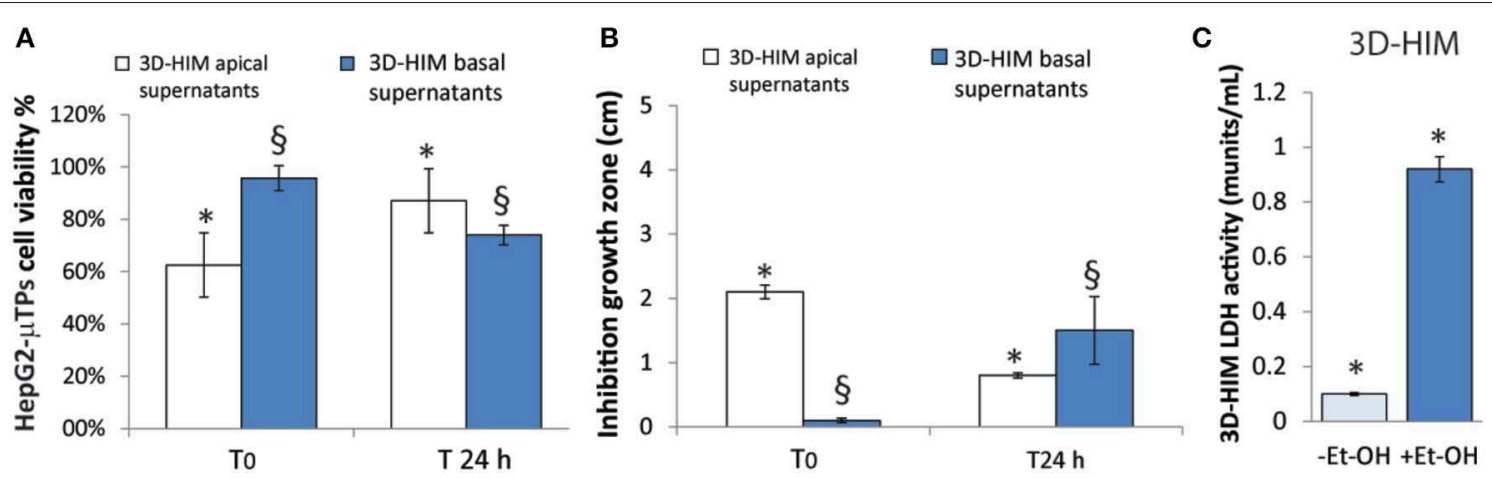

FIGURE 4 | 3D-HIM-metabolized Et-OH effect on HepG2- $\mu$ TPs cytotoxicity and L. rhamnosus growth and Et-OH damage on 3D-HIM. MTT cell viability assay displays indirect estimation of the indigested Et-OH treating HepG2- $\mu$ TPs with 3D-HIM supernatants (3D-HIMapical and -basal supernatants) at two different time points ( $\mathrm{T}_{0}$ and $24 \mathrm{~h})\left({ }^{*} p<0.001 ;{ }^{\S} p<0.05\right)$ (A); Zone of Inhibition Test shows the antimicrobial activity of the indigested Et-OH treating HepG2- $\mu$ TPs with 3D-HIM supernatants (3D-HIM $M_{\text {apical }}$ and -basal supernatants) on L. rhamnosus growth at two different time points ( $\mathrm{T}_{0}$ and $\left.24 \mathrm{~h}\right)\left({ }^{*} p<0.001 ;{ }^{\S} p<0.05\right)(\mathbf{B}) ; \mathrm{LDH}$ assay indicates the 3D-HIM toxicity after $400 \mathrm{mM}$ Et-OH treatment (C) $\left.{ }^{*} p<0.01\right)$.

junction between the Caco-2 cells, determining an increase in barrier permeability (Figures 5a,b, respectively). Quantitative immunofluorescence analysis showed that the number of the tight junctions found on the untreated samples (189 \pm 14.8 , number of tight junctions per field) was higher compared to Et$\mathrm{OH}$-treated 3D-HIM ( $82 \pm 8.01$, number of tight junctions per field) (Figure 5c). Further, immunofluorescence staining for the Laminin $\mathrm{V}$ along the interface between stroma and epithelium indicated the presence of an intact basement membrane with a polarized epithelium on the untreated sample (-Et-OH, Figure 5d). In contrast, Et-OH treatment compromised the basement membrane integrity, as shown by pixelated signal of Laminin $\mathrm{V}$ and revealed the separation of the supra-basal epithelium from the basal lamina, which ultimately could cause the epithelium rupture (Figure 5e). In addition, an altered epithelial organization was also detected in Et-OH-treated 3D-HIM. TEER measurements were performed in order to confirm the damage at epithelium permeability. In agreement with immunofluorescence results, the untreated 3D-HIM (Et-OH) expressed higher TEER values (4.72 $\mathrm{k} \Omega$ ) compared to Et-OH-treated 3D-HIM (43.4 $\Omega$ ) (Figure 5f), confirming that Et-OH treatment affected paracellular junctions. On the contrary, the capacitance measurements on untreated and treated samples resulted comparable (1.1 and $1.34 \mu \mathrm{F}$, respectively), suggesting that the membranes of the epithelial cells remain intact. Furthermore, in order to evaluate the mucus secretion on untreated (-Et-OH) and Et-OH-treated samples (+Et$\mathrm{OH}$ ), Alcian blue staining was performed. 3D-HIM respond to Et-OH treatment by overproducing mucus on Et-OHtreated 3D-HIM compared with the untreated one (Figures 5g,h, respectively). Quantification analysis of the Alcian blue images further confirmed the qualitatively observation of the histological images (Figure 5i). Moreover, analysis of the composition and architecture of the ECM was performed. Figure 6 reported the Second Harmonic Generation ( $\mathrm{SHG}$ ) images rising from newly formed collagen signal on the untreated $(-\mathrm{Et}-\mathrm{OH}$, Figure 6a) and Et-OH-treated 3D-HIM (+Et-OH, Figure 6b).
The SHG images were exploited to estimate the CF, the CAD, and the correlation length of collagen network in the ECM (Figures 6c-e). Statistically significative differences were found between the values of untreated and Et-OH-treated samples. Both CF and CAD were affected by Et-OH treatment, 43.64 $\pm 6.6 \%$ vs. $32.48 \pm 5.0 \%$, the former, and $31.00 \pm 7.6$ vs. $24.43 \pm 3.0 \mathrm{a} . \mathrm{u}$, the latter. In addition, the correlation length highlights differences in collagen texture, reporting a value for untreated samples of $52.34 \pm 2.50$ a.u. and a value for Et$\mathrm{OH}$-treated samples of $37.40 \pm 2.10$ a.u (Figure 6e). Lastly, Et$\mathrm{OH}$ treatment induced MMP-9 overexpression, as reported in Figures 6f,g.

\section{Protective Role of 3D-HIM on Et-OH-Induced Hepatic Cytotoxicity in InLiver-OC}

In order to determine the intestine contribution to Et$\mathrm{OH}$ metabolism, $400 \mathrm{mM}$ Et-OH was administrated in three configurations of the device: Control group samples (untreated), Et-OH-treated Liver-OC (HepG2- $\mu$ TPs without 3D-HIM), and Et-OH-treated InLiver-OC (device with both HepG2- $\mu$ TPs and 3D-HIM). Histological characterization of untreated samples using $\mathrm{H} \& \mathrm{E}$ staining displayed a homogenous cell distribution around the microbeads surface of the HepG2- $\mu$ TPs with the typical liver-like histotypic features, such as cuboidal hepatocyte cell shape with tight cell-cell contacts. In the Liver-OC configuration, HepG2- $\mu$ TPs, although preserving the 3D epithelial organization, showed a high amount of lipid accumulation, as indicated by the black arrowheads. In contrast, in InLiver-OC configuration, HepG2- $\mu$ TPs showed a small amount of lipid accumulation with a well-polarized epithelium (Figures 7a-c). Immunofluorescence staining showed that the Claudin-1 expression was higher in control groups than in Liver-OC, in which a completely damaged tight junction network was detected (Figures $\mathbf{7 d , e}$, respectively). In contrast, a continuous network of tight junction was found in some 


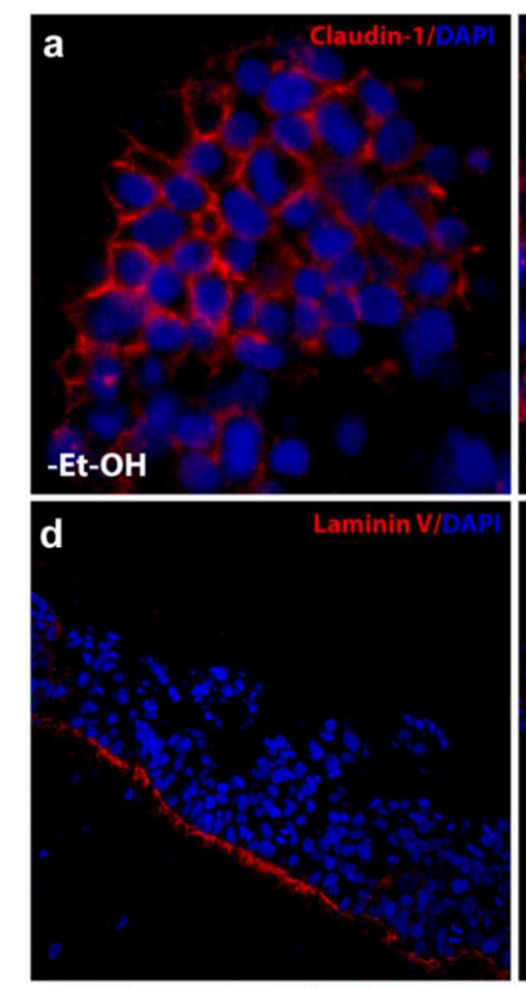

g

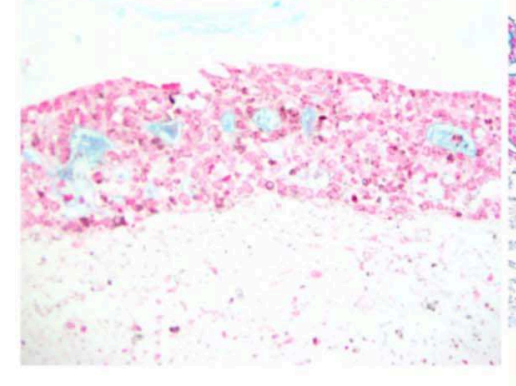

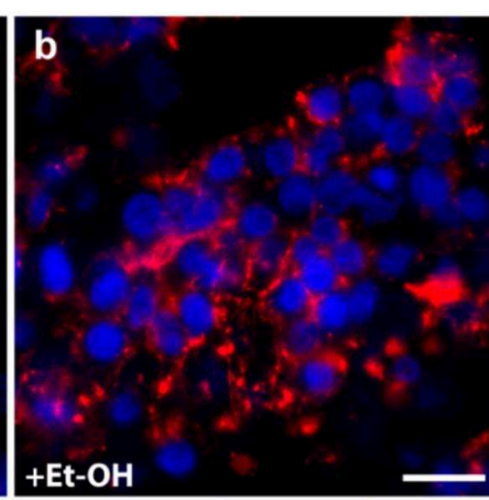
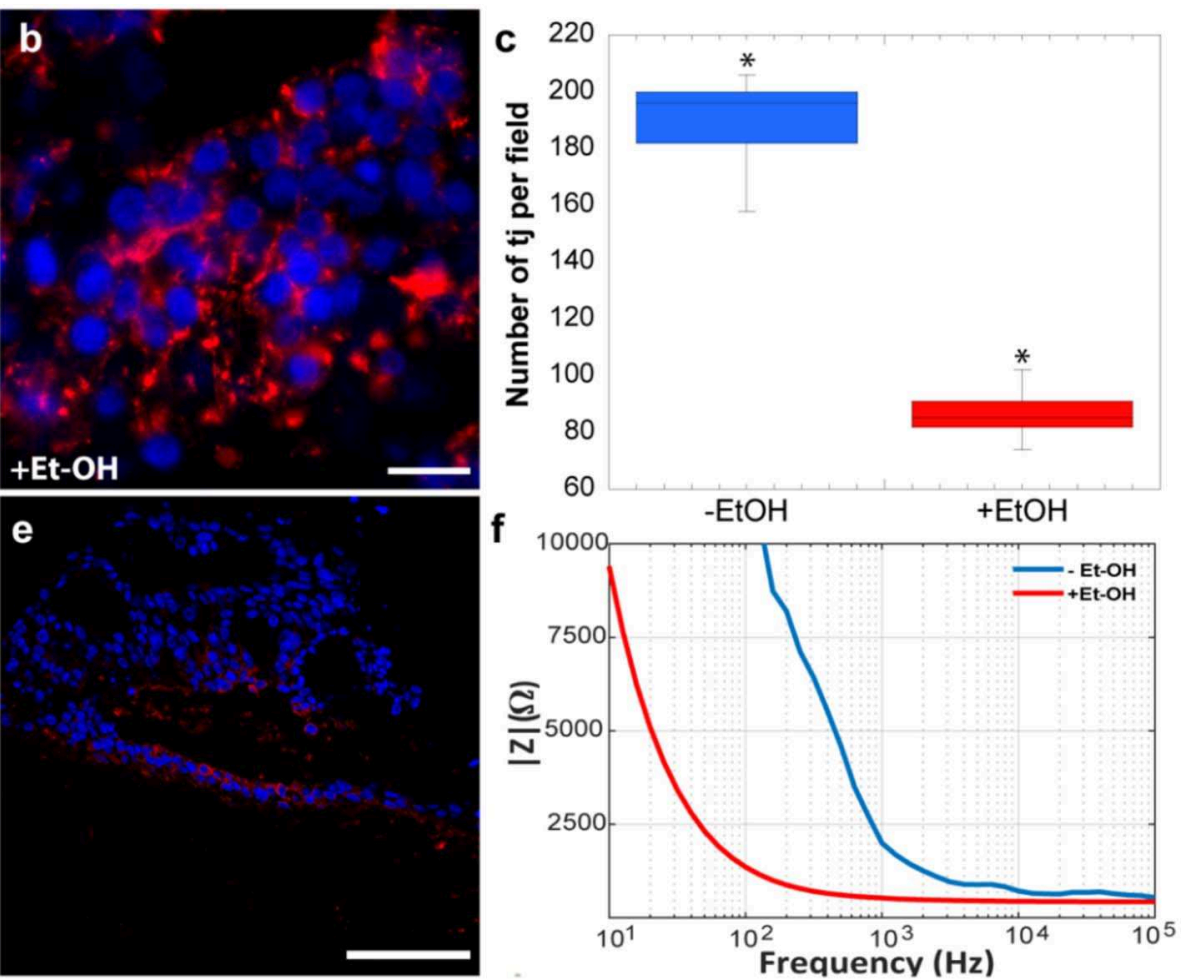

h

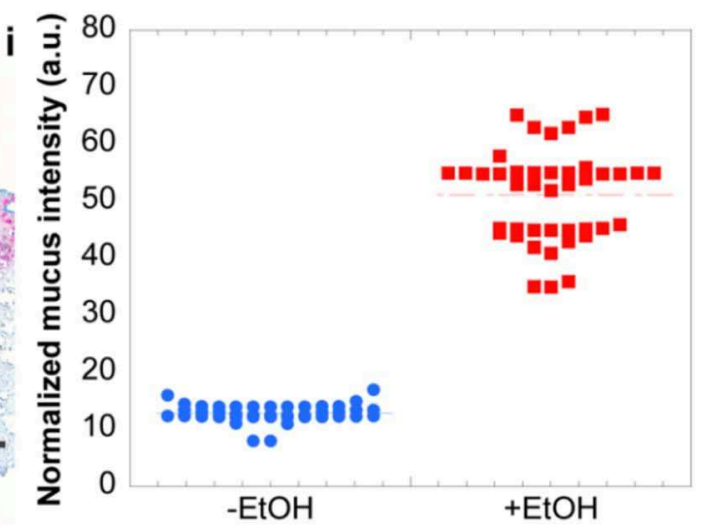

FIGURE 5 | Et-OH-induced 3D-HIM hyper-permeability at epithelial level. Comparison of the expression of the intestinal epithelial tight junction cell marker (Claudin-1) on untreated (-EtOH) 3D-HIMs (a) and $400 \mathrm{mM}$ Et-OH-treated (+Et-OH) 3D-HIMs (b); scale bar $50 \mu \mathrm{m}$. Quantitative analysis of tight junction per field ( $\left.{ }^{\star} p<0.05\right)$ (c); confocal microscope analysis of immunofluorescence staining shows the Laminin $\mathrm{V}$ signal on untreated (-EtOH) 3D-HIMs (d) and 400 mM Et-OH treated (+Et-OH) 3D-HIMs (e); cell nuclei stained with DAPI scale bar $75 \mu \mathrm{m}$; Quantitative analysis of TEER values (f); Alcian blue-stained cross-sections reveals the mucus deposition in untreated (-EtOH) 3D-HIMs (g) and 400 mM Et-OH-treated (+Et-OH) 3D-HIMs (h); scale bar $100 \mu$ m; Quantitative analysis of Alcian blue-stained samples reveals the mucus production (i). All the experiments were performed in triplicate $(n=3)$; values represent the mean and the standard deviation.

area of HepG2- $\mu$ TPs in InLiver-OC (Figure 7f). Moreover, in order to better characterize the liver functionality, HepG2$\mu \mathrm{TPs}$ were stained with P-Glycoprotein (P-gp) antibody-a membrane's protein that guides the transport of the substance across the epithelium-and a qualitative analysis of the Pgp expression for untreated samples, Liver-OC, and InLiverOC was performed, as highlighted in Figures $7 \mathbf{g}$-i. Specifically, as depicted in Figure $\mathbf{7 g}$, the formation of canalicular-like structures was evidenced in untreated samples. However, a slight signal of P-gp was detected in Liver-OC, which indicated no assembly of the membrane protein (Figure $7 \mathbf{h}$ ) in this condition, while the InLiver-OC showed higher expression of this protein, well-organized both in the core and on the external surface of the microtissue (Figure 7i). Furthermore, in order to determine the lipid accumulation on HepG2$\mu$ TPs, oil red staining was assessed directly into the biochip (Figure S5) or cross-sections after withdrawing the sample from the device (Figures $\mathbf{7 j - 1}$ ). In particular, untreated samples 

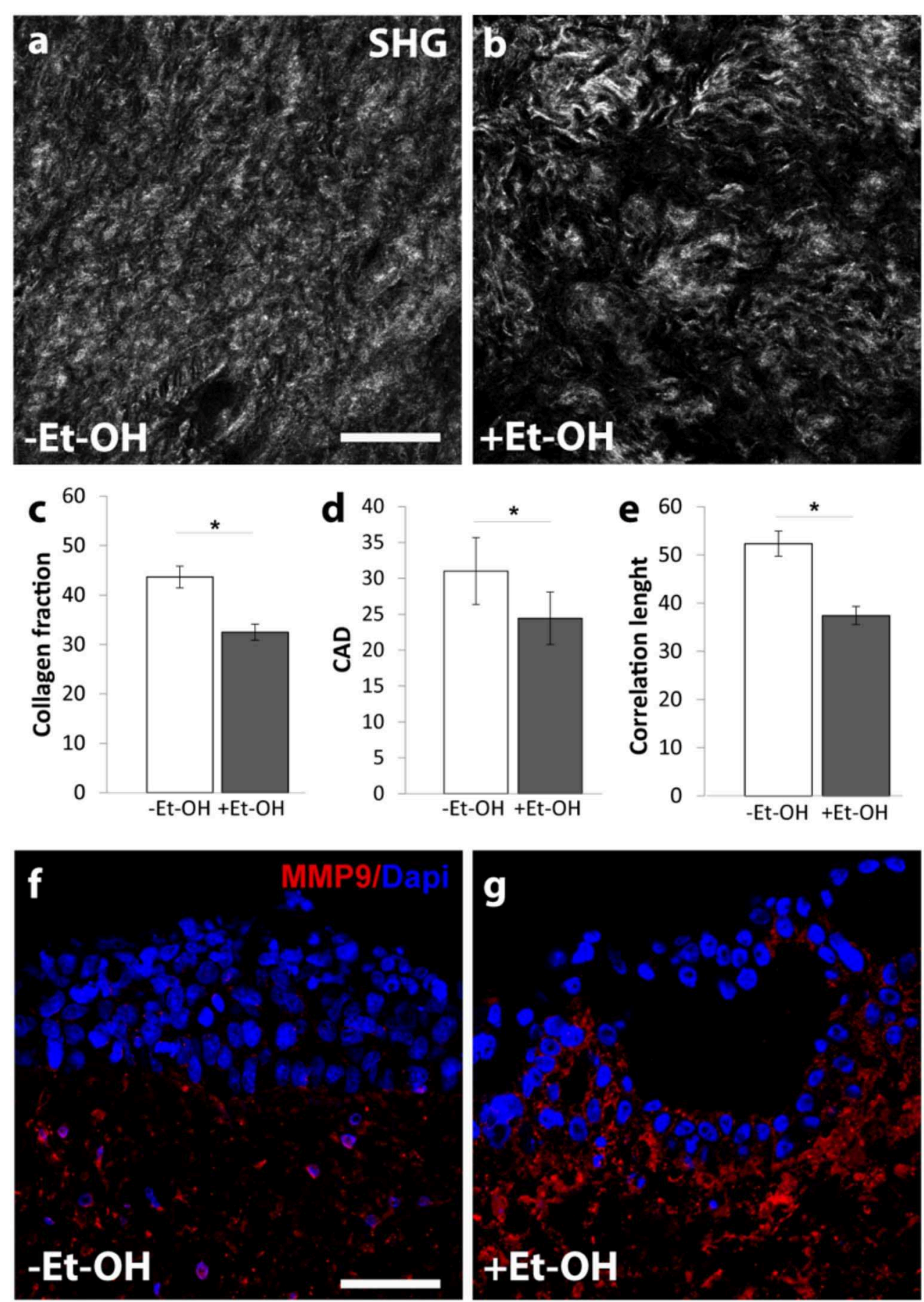

FIGURE 6 | Et-OH-induced 3D-HIM stromal injury. Representative high magnification SHG images (gray scale) of the auto-produced collagen in untreated (-EtOH) 3D-HIMs (a) and 400 mM Et-OH-treated (+Et-OH) 3D-HIMs (b); scale bar $75 \mu \mathrm{m}$; (c) Collagen fraction, (d) CAD and (e) GLCM analysis in untreated (-EtOH) and $400 \mathrm{mM}$ Et-OH-treated $(+\mathrm{Et}-\mathrm{OH}) 3 \mathrm{D}-\mathrm{HIMs}\left({ }^{*} \mathrm{P}<0.001\right)$; immunofluorescence images indicate different protein expression stained in red (MMP9) on untreated (-EtOH) $3 \mathrm{D}-\mathrm{HIMs}(\mathbf{f})$ and $400 \mathrm{mM}$ Et-OH-treated $(+\mathrm{Et}-\mathrm{OH}) 3 \mathrm{D}-\mathrm{HIMs} \mathbf{( g )}$; scale bar is $75 \mu \mathrm{m}$; DAPI (blue fluorescence). All the experiments were performed in triplicate $(n=3)$; values represent the mean and the standard deviation.

showed a small amount of lipid accumulation. In contrast, high amount of lipid drops (stained in red, black arrowheads) were displayed in Liver-OC, and a small quantity of lipid drops was found in InLiver-OC cross-sections, as reported in Figures 7j-1.
Finally, ROS were found to be over-expressed in Liver-OC compared to untreated and InLiver-OC. Figure S6 shows that the ROS expression on the Liver-OC was higher than on the InLiver-OC. 


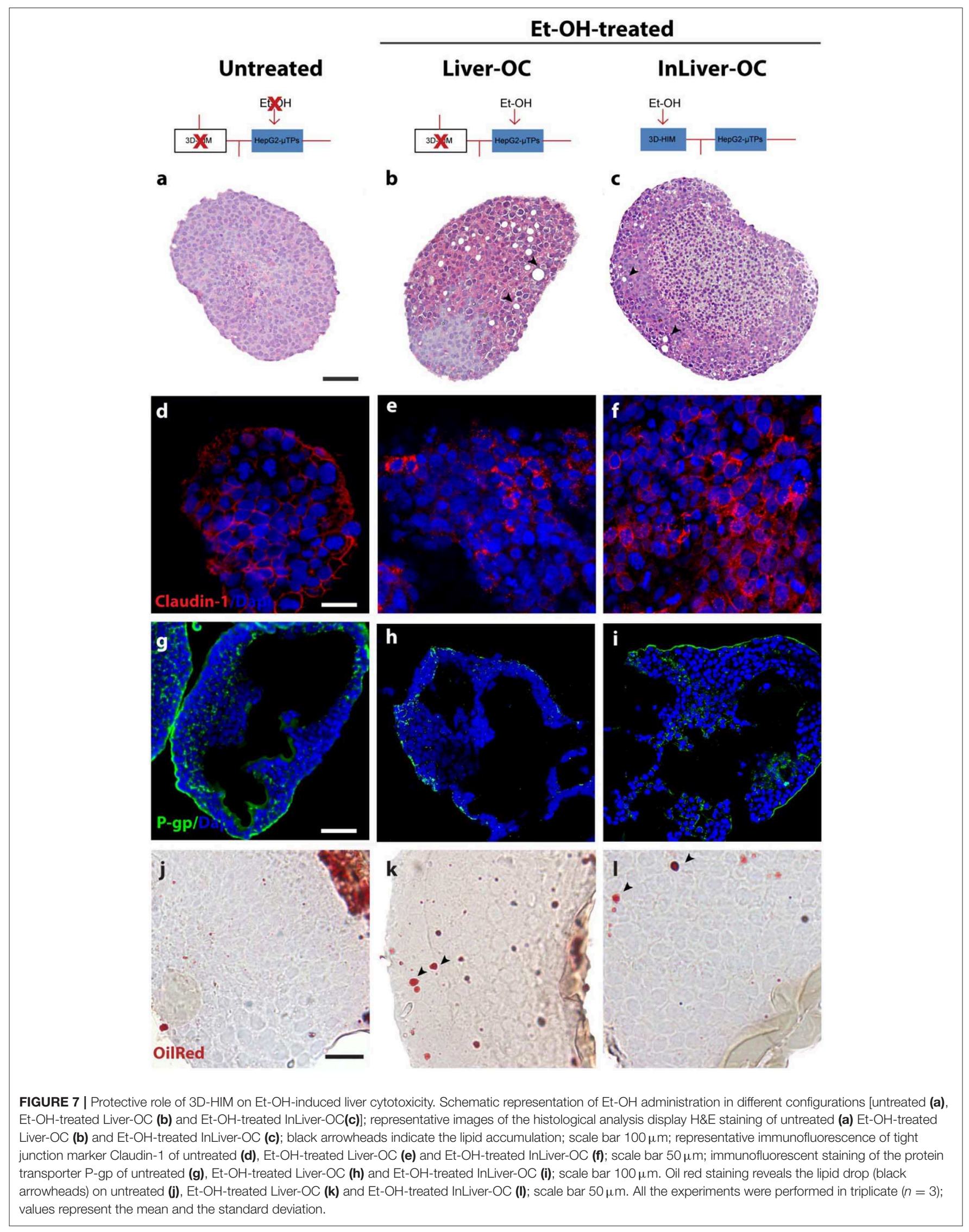




\section{Synergic Response of 3D-HIM and HepG2- $\mu$ TPs After Et-OH Administration in InLiver-OC}

In order to determine the 3D-HIM as well as the HepG2$\mu$ TPs response to the Et-OH treatment in the device, three configurations were explored: In-OC (device loaded with 3D-HIM without HepG2- $\mu$ TPs), Liver-OC (device loaded with HepG2- $\mu$ TPs without 3D-HIM), and InLiver-OC (device with both HepG2- $\mu$ TPs and 3D-HIM). In the InOC configuration, the tissue supernatants were collected from the apical compartment of the Intestine $e_{c}$ and from the sampling channel between the Intestine ${ }_{c}$ and Liver $_{c}$. In the Liver-OC configuration, the tissue supernatants were collected downstream to the Liver ${ }_{c}$. And, in the InLiver-OC configuration, the tissue supernatants were collected only downstream to the Liver $_{c}$ (Figure 8A). The detection of ADH activity was used as indication of acute cell damage (Figure 8B). As deduced from the results obtained after treatment with $400 \mathrm{mM}$ Et-OH, a high amount of $\mathrm{ADH}$ was detected in the Intestine $_{\text {apical }}$ $\left(\mathrm{In}_{\text {apical }}\right)(2.42 \pm 0.65 \mathrm{mU} / \mathrm{mL})$, indicating the enzyme activity of 3D-HIM. In addition, a slight but significant release of $\mathrm{ADH}$ was revealed in the sampling channel $\left(\mathrm{In}_{\text {basal }}\right)(1.33 \pm$ $0.32 \mathrm{mU} / \mathrm{mL})$. Further, a higher amount of ADH $(4.27 \pm 0.42$ $\mathrm{mU} / \mathrm{mL}$ ) was detected downstream to the Liver $_{\mathrm{c}}$ in the Liver-OC configuration, mimicking what occurs during acute alcoholic liver injury. At last, InLiver-OC showed the highest ADH value $(7.2 \pm 0.56 \mathrm{mU} / \mathrm{mL})$ compared to the other configuration, suggesting a synergic activity of both intestinal and hepatic tissue co-cultured into the device. Meanwhile, experiments were performed to evaluate whether the Et-OH injury induced MDRs gene expression modulation. 3D-HIM as well as the HepG2- $\mu$ TPs samples cultured in Liver-OC or InLiver-OC were processed to quantify the MDR gene expression by means of molecular analysis. The results indicated that MDR4 expression on 3D-HIM decreased after Et-OH injury $(5.3 \pm 0.6$ a.u. $)$ compared to untreated samples $(7.9 \pm 2.4$ a.u.) (Figure $8 \mathrm{C})$. Likewise, Et-OH-treated HepG2- $\mu$ TPs cultured in Liver-OC displayed low values of the MDR4 gene expression (3.8 \pm 0.9 a.u.) compared to untreated samples $(7.2 \pm 1.5)$. On the other hand, when HepG2- $\mu$ TPs were cultured in InLiver-OC, they displayed higher values in the MDR4 gene expression (8.1 \pm 1.6 a.u.) compared to Et-OH-treated Liver-OC, suggesting a protective action of intestine on hepatic function. Furthermore, an increase of P-gp gene expression $(9.8 \pm 2.6)$ was revealed on 3D-HIM after Et-OH injury, in agreement with the in vivo situation. P-gp gene expression slightly increased on Et-OHtreated Liver-OC $(7.9 \pm 1.7)$ but reached higher values on Et-OH treated InLiver-OC $(9.2 \pm 2.8)$ compared to untreated samples (7.5 \pm 1.7 ) (Figures 8D,E). Lastly, the biomass production after $400 \mathrm{mM}$ Et-OH treatment was measured, showing the amount of cell debrides collected in the tissue supernatants. In particular, Et-OH-treated Liver-OC showed highest biomass compared to the other conditions, indicating the major cell debrides accumulation due to the cell damage in this configuration. The biomass quantification of InLiver-OC Et-OH-treated samples correspond to the sum of the two organs contributions: $20 \%$ of
Liver-OC debrides (extrapolated from the HepG2- $\mu$ TPs viability assay) and In-OC debrides (Figure S7).

\section{DISCUSSION}

It is widely recognized that the intestinal and hepatic firstpass metabolism may depress extensively the bioavailability of drugs and xenobiotic compounds (Rowland, 1972), highlighting the urgent need for robust in vitro models able to recapitulate this process to support drug and nutraceutics discovery and testing. Here, an integrated microfluidic intestine-liver device (InLiver-OC) has been proposed to investigate the in vivo route of orally ingested Et-OH by connecting directly the two main organs (intestine and liver) involved in the first-pass metabolism. The InLiver-OC device was designed in order to allow the inter-organs crosstalk recapitulating the key features of human intestine-liver physiology and functionality. A previously established modular tissue assembling approach to fabricate the intestinal stroma (3D-ISM) composed by H-InMyoFibs embedded in their own ECM has been exploited, and then fullthickness 3D-HIM has been obtained after Caco-2 seeding and culture (De Gregorio et al., 2018a,b). Then, 3D HepG2- $\mu$ TPs have been obtained by using dynamic cells seeding in a spinner flask bioreactor (Corrado et al., 2019). The microfluidic device (InLiver-OC) was designed to host the two 3D physiologically relevant in vitro models (3D-HIM and HepG2- $\mu$ TPs) and to provide a direct link between them by connecting in a continuous fluid stream the basal side of the Intestine $e_{c}$ and the Liver ${ }_{c}$. As first issue, the optimization of culture media mixture to perform the organ-on-chip co-culture was faced. As reported in literature, some researchers solved the problems by using multi-organon-chip composed by independent modules and transferring the media from one organ module to the subsequent in a physiological sequence, which allows each organ module to be operated independently (Tsamandouras et al., 2017). However, this method leads the dilution of the culture media from one module to another, avoiding the metabolic waste accumulation, which could lead to the loss of metabolic products that are crucial for experimental purposes. In this perspective, in our InLiver-OC, the modules are directly connected and the culture medium opportunely optimized. Et-OH was selected as harmful stimulus, and the Et-OH concentration to mimic alcoholinduced liver damage was selected by performing cytotoxicity assay under different Et-OH concentration on HepG2 cells in both $2 \mathrm{D}$ and $3 \mathrm{D}$ configurations. The results indicated that Et-OH significantly increases the death rate in $2 \mathrm{D}$ HepG-2 monolayer samples compared to 3D HepG2- $\mu$ TPs that maintained high cell vitality, suggesting that the $3 \mathrm{D}$ configuration was more reliable than 2D for cytotoxicity assay (Edmondson et al., 2014). In general, the main issue in reproducing the first-pass mechanism is the maintenance of a selective transport route from the apical to the basal side of the Intestine ${ }_{c}$. To achieve this, we fabricated a sealed chamber by using a PDMS gasket and a PMMA cylinder accommodated over the 3D-HIM. As it is well-known, once consumed, alcohol is absorbed mainly in the upper intestinal tract by diffusion and then enters the liver 
A

In-OC

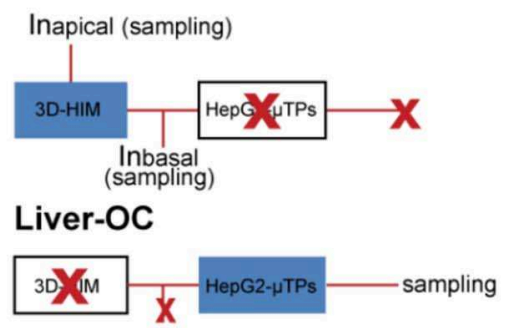

InLiver-OC

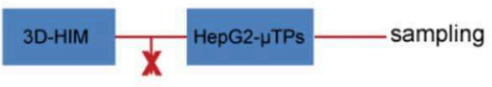

B

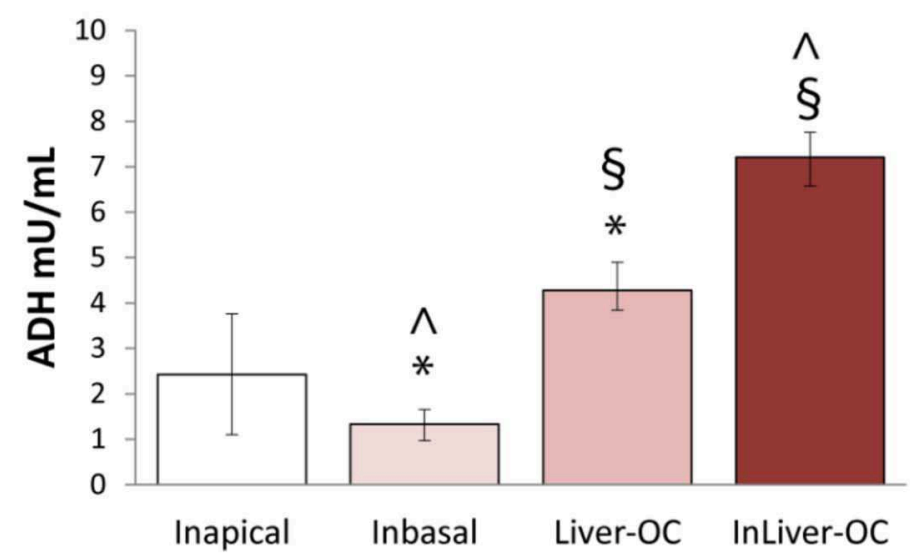

C

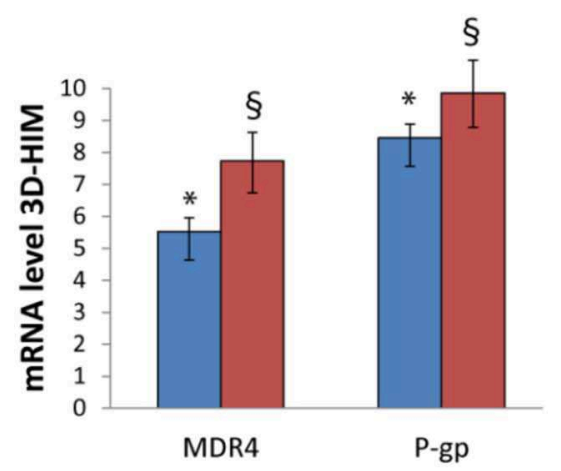

D

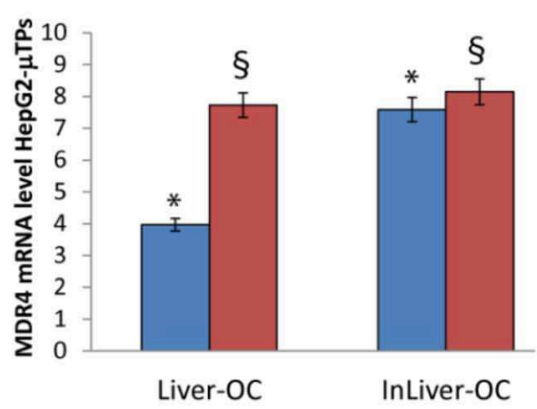

E

FIGURE 8 | Synergic response of 3D-HIM and HepG2- $\mu$ TPs after Et-OH administration. Overview of the experimental design: In-OC (device loaded with 3D-HIM without HepG2- $\mu$ TPs), Liver-OC (device loaded with HepG2- $\mu$ TPs without 3D-HIM) and InLiver-OC (device with both HepG2- $\mu$ TPs and 3D-HIM); Inapical Sampling

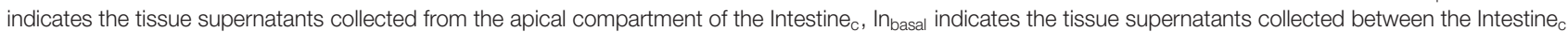
and Liver $_{\mathrm{C}}$ and sampling indicates the tissue supernatants collected downstream to the Liver ${ }_{\mathrm{C}}(\mathbf{A})$; quantification analysis of ADH activity in the Intestine apical $\left._{\text {( }} \mathrm{I}_{\text {apical }}\right)$, in the sampling channel (In basal), Liver-OC, and Liver-OC; " $\wedge$ " indicates that the group samples Inbasal and InLiver-OC are considered to be statistically significant $(p<$ $0.001)$ (B); quantification analysis of P-gp and MDR4 genes expression on untreated $(-\mathrm{Et}-\mathrm{OH})$ and $\mathrm{Et}-\mathrm{OH}$-treated $(+\mathrm{Et}-\mathrm{OH}) 3 \mathrm{D}-\mathrm{HIM}\left({ }^{*} p<0.0001 ;{ }^{\S} p<0.05\right)(\mathbf{C})$; MDR4 (D); and P-gp (E) gene expression of untreated (-Et-OH) and Et-OH-treated (+Et-OH) HepG2- $\mu$ TPs $\left({ }^{*} p<0.001 ;{ }^{\S} p<0.05\right)$ in the two different device configurations: Liver-OC or InLiver-OC. All the experiments were performed in triplicate $(n=3)$; values represent the mean and the standard deviation.

via the portal vein (Bishehsari et al., 2017). However, although the majority of alcohol metabolism occurs in hepatocytes, the enzymes involved in the oxidative or non-oxidative metabolism of alcohol are also present in the intestinal mucosa (Cederbaum, 2012). Mounting evidence suggests that alcohol, particularly if consumed chronically or in larger amounts, induces a deleterious effect at the intestinal district that in turn promotes inflammation through various pathways, including changes in intestinal microbiota composition and function (Mutlu et al., 2012; Engen et al., 2015), increased permeability of the intestinal mucosa (Günzel and Yu, 2013), and disruptions of the immune system of the intestinal mucosa (Bishehsari et al., 2017), as well. With this perspective, we deeply investigated the 3D$\mathrm{HIM}$ response to Et-OH treatment by analyzing supernatants from apical and basal side of 3D-HIM. Surprisingly, we found that Et-OH is partly digested by 3D-HIM after $24 \mathrm{~h}$, protecting HepG2- $\mu$ TPs from cell mortality. In addition, in line with the literature reporting a correlation between alcohol and dysbiosis (Canesso et al., 2014), our results demonstrated the antibacterial activity on the L. rhamnosus strain growth of supernatants withdrawn from $3 \mathrm{D}-\mathrm{HIM}_{\text {apical }}$ at $\mathrm{T}_{0}$ due to the presence of undigested Et-OH and of supernatants from $3 \mathrm{D}-\mathrm{HIM}_{\text {apical }}$ and -basal at $24 \mathrm{~h}$ due to the presence of Et-OH or its metabolites. Alcohol has been known for a long time to interfere with the absorption of several nutrients and to lead to small intestinal mucosal damage, thereby contributing to the increase of the intestinal trans-epithelial as well as para-cellular permeability by acting on the tight junction complex (Bode and Bode, 2003). Intestinal epithelial tight junctions' integrity is fundamental in the studies of absorption and permeation of compounds, because 
they form a physical barrier to the diffusion of macromolecules (Günzel and Yu, 2013). Previous studies demonstrated that the acetaldehyde, rather than Et-OH, can cause the disruption of tight junctions in Caco-2 monolayer (Atkinson and Rao, 2001). As predictable, the 3D-HIM-epithelial damage was found after Et-OH administration in InLiver-OC by analyzing the Claudin-1 signal, a transmembrane protein of the tight junctions, that form a barrier to macromolecular diffusion (Günzel and $\mathrm{Yu}, 2013)$. Specifically, the Et-OH-treated 3D-HIM presented a lower number of tight junctions with less organized protein structure. In addition, the TEER measurements on Et-OHtreated 3D-HIM confirmed the Et-OH deleterious effect on the epithelial barrier showing a very low TEER value compared to untreated samples. Further, the acute alcohol administration elicits basement membrane alterations determining in severe cases the separation of the epithelium from the basal lamina with the formation of sub-epithelial blisters, which ultimately cause the epithelium rupture (Singer and Brenner, 2006). In agreement, we found that in Et-OH-treated InLiver-OC, the 3D-HIM showed a basement membrane impairment reported as pixelated immunofluorescence signal of the main protein of basal lamina (Laminin V) (Haas et al., 2001). In addition, in Et-OH-treated 3D-HIM, a high quantifiable amount of mucus was produced, due to the self-defense to the high concentration of the Et-OH and its metabolites. This is in agreement with previous studies showing that chronic alcohol feeding increased mucin production in the small intestine due to alcohol-induced qualitative changes (Valatas and Kolios, 2009). However, the damage induced to the small intestine by the Et-OH involves not only the epithelial layer but also affects the stromal compartment (Casini et al., 1999). From this perspective, the availability of a 3D-HIM provided with an endogenous stroma allows to assess the complex response of the small intestine to Et-OH, otherwise not observable with the over-simplistic existing intestinal mucosa model. SHG microscopy on 3D-HIM provided high-resolution $3 \mathrm{D}$ images of collagen fibers in thick samples without the need for sample staining and processing and allowed us to detect the non-centrosymmetrical structure of fibrillar collagen and the textural change at stromal level resulting from Et$\mathrm{OH}$ treatment (De Gregorio et al., 2019). The results indicated that Et-OH-treated 3D-HIM experienced a decrease of both the total collagen amount and of the degree of the collagen assembly, as well as a collagen stretching in the intestinal mucosa. It is reasonable to assume that ECM remodeling contributes to Et-OH intestinal mucosa injury. In terms of liver damage due to Et-OH administration we assessed the role of intestine in reducing the toxic effects by analyzing morphological and functional hepatic markers in InLiver-OC vs. Liver-OC. Our results showed a protective role of intestine able to impair the lipid accumulation and maintain quite intact the tight junction's network in Et-OH-treated HepG2- $\mu$ TPs cultured in InLiverOC. Some in vivo studies suggested that intestinal drug efflux transporters are involved in pharmacokinetic alterations caused by chronic alcohol exposure (Artursson and Karlsson, 1991; Sambuy et al., 2005). Here, we reported a strong increase of Pgp gene expression on 3D-HIM after Et-OH injury according to the in vivo situation. High value of $\mathrm{P}-\mathrm{gp}$ gene expression in Et-OH-treated HepG2- $\mu$ TPs in InLiver-OC indicate once again the preservation of biochemical liver function from intestine. Furthermore, in terms of protein expression, we found the correct signal of P-gp that pointed out the formation of canalicular-like structures in InLiver-OC. In addition, the high value of MDR4 gene expression indicated a physiological liver protection from toxic accumulation of bile acids and a low cytosolic ROS production in Et-OH treated HepG2- $\mu$ TPs cultured in InLiver-OC samples, corroborating once again the intestine prevention to the liver injury. Even if the majority of alcohol metabolism in humans occurs in the hepatocytes cells in the liver, some enzymes involved in the oxidative metabolism of alcohol such as alcohol dehydrogenase (ADH) are also present in the intestinal mucosa. In line with the in vivo situation, $\mathrm{ADH}$ was detected in the $3 \mathrm{D}$-HIM as well as HepG2- $\mu$ TPs, indicating the enzyme activity of InLiver-OC after Et-OH treatment. Liver-OC also showed high amount of $\mathrm{ADH}$ indicating acute alcoholic liver injury. These data confirmed the synergic contribution of both tissues when co-cultured into InLiver-OC together. Lastly, it should also be noted that the biomass production after Et-OH treatment indicated the comparable debris accumulation when single tissues culture (InOC or Liver-OC) or co-culture (InLiver-OC) were performed in the device. These results suggested that the culture medium guaranteed the physiological function of both intestine and hepatic equivalent tissues and did not induce hepatic damage due to intestinal metabolic waste accumulation in InLiver-OC. Taken together, these results point out the stromal and epithelial damage of the 3D-HIM under orally administrated xenobiotic and the protective intestinal role in attenuating the Et-OH-induced liver cytotoxicity.

\section{CONCLUSION}

In conclusion, in this study, we developed a microfluidic InLiverOC with the aim of reproducing the first pass metabolism. We have observed the intestine-liver crosstalk in the metabolic and absorptive properties. By using the Et-OH as a harmful stimulus, we have shown that this device can be used as a tool to reproduce the first-pass metabolism of drugs and xenobiotics. Our proposed model is a more predictive platform than the $2 \mathrm{D}$ cell culture, resembling the physiological way route of the in vivo first-pass metabolism. It is also suitable to study the interaction of the gastrointestinal tract, the effects of nutraceutical substances, and the uptake of compounds. InLiver-OC can be adapted to co-culture human gut microbiome to provide a versatile platform to investigate hostmicrobiome interaction in a multi-organs platform. Finally, this microdevice is expected to reduce the number of drug candidates and accelerate the pre-clinical screening process reducing animal testing.

\section{DATA AVAILABILITY STATEMENT}

The datasets generated for this study are available on request to the corresponding author. 


\section{AUTHOR CONTRIBUTIONS}

GI and PN conceived the idea and critically revised the manuscript with input from the entire team. VD, GI, and FU worked on the study conception and design, analyzed and interpreted the data, and drafted the manuscript. VD and MT carried out biological experiments. VR performed electrophysiological measurements. BC designed and microfabricated the device. FU performed fluidodynamic simulations experiments. All authors have read and approved the final draft.

\section{REFERENCES}

Artursson, P., and Karlsson, J. (1991). Correlation between oral drug absorption in humans and apparent drug permeability coefficients in human intestinal epithelial (Caco-2) cells. Biochem. Biophys. Res. Commun. 175, 880-885. doi: 10.1016/0006-291X(91)91647-U

Atkinson, K., and Rao, R. (2001). Role of protein tyrosine phosphorylation in acetaldehyde-induced disruption of epithelial tight junctions. Am. J. Physiol. Gastrointest. Liver Physiol. 280, G1280-G1288. doi: 10.1152/ajpgi.2001.280.6.G1280

Bhatia, S. N., and Ingber, D. E. (2014). Microfluidic organs-on-chips. Nat. Biotechnol. 32, 760-772. doi: 10.1038/nbt.2989

Bishehsari, F., Magno, E., Swanson, G., Desai, V., Voigt, R. M., Forsyth, C. B., et al. (2017). Alcohol and gut-derived inflammation. Alcohol Res. 38, 163-171.

Bode, C., and Bode, J. C. (2003). Effect of alcohol consumption on the gut. Best Pract. Res. Clin. Gastroenterol. 17, 575-592. doi: 10.1016/S1521-6918(03)00034-9

Canesso, M. C. C., Lacerda, N., Ferreira, C., Gonçalves, J., Almeida, D., Gamba, C., et al. (2014). Comparing the effects of acute alcohol consumption in germ-free and conventional mice: the role of the gut microbiota. BMC Microbiol. 14:240. doi: 10.1186/s12866-014-0240-4

Casini, A., Galli, A., Di Lollo, S., Orsini, B., Arganini, L., Jezequel, A. M., et al. (1999). Ethanol-induced alterations of matrix network in the duodenal mucosa of chronic alcohol abusers. Virchows Arch. 434, 127-135. doi: $10.1007 / \mathrm{s} 004280050316$

Cederbaum, A. I. (2012). Alcohol metabolism. Clin. Liver Dis. 16, 667-685. doi: 10.1016/j.cld.2012.08.002

Chen, H. J., Miller, P., and Shuler, M. L. (2018). A pumpless body-on-a-chip model using a primary culture of human intestinal cells and a $3 \mathrm{D}$ culture of liver cells. Lab Chip 18, 2036-2046. doi: 10.1039/C8LC00111A

Chen, W. L., Edington, C., Suter, E., Yu, J., Velazquez, J. J., Velazquez, J. G., et al. (2017). Integrated gut/liver microphysiological systems elucidates inflammatory inter-tissue crosstalk. Biotechnol. Bioeng. 114, 2648-2659. doi: 10.1002/bit.26370

Choe, A., Ha, S. K., Choi, I., Choi, N., and Sung, J. H. (2017). Microfluidic Gutliver chip for reproducing the first pass metabolism. Biomed. Microdevices 19:4. doi: 10.1007/s10544-016-0143-2

Corrado, B., De Gregorio, V., Imparato, G., Attanasio, C., Urciuolo, F., and Netti, P. A. (2019). A three-dimensional microfluidized liver system to assess hepatic drug metabolism and hepatotoxicity. Biotechnol. Bioeng. 116, 1152-1163. doi: $10.1002 /$ bit. 26902

Council, N. R. (2007). Toxicity Testing in the 21st Century: A Vision and a Strategy. Washington, DC: National Academies Press.

De Gregorio, V., Corrado, B., Sbrescia, S., Sibilio, S., Urciuolo, F., Netti, P. A., et al. (2019). Intestine-on-chip device increases ECM remodeling inducing faster epithelial cell differentiation. Biotechnol. Bioeng. 117, 556-566. doi: 10.1002/bit.27186

De Gregorio, V., Imparato, G., Urciuolo, F., and Netti, P. A. (2018a). 3D stromal tissue equivalent affects intestinal epithelium morphogenesis in vitro. Biotechnol. Bioeng. 115, 1062-1075. doi: 10.1002/bit.26522

De Gregorio, V., Imparato, G., Urciuolo, F., and Netti, P. A. (2018b). Micro-patterned endogenous stroma equivalent induces polarized crypt-villus

\section{ACKNOWLEDGMENTS}

The authors thank Dr. La Rocca Alessia for her precious contribution during the experimental phase. The authors thank Dr. Sibilio Sara for her contribution in Comsol studies.

\section{SUPPLEMENTARY MATERIAL}

The Supplementary Material for this article can be found online at: https://www.frontiersin.org/articles/10.3389/fbioe. 2020.00163/full\#supplementary-material

architecture of human small intestinal epithelium. Acta Biomater. 81, 43-59. doi: 10.1016/j.actbio.2018.09.061

de Sousa, I. P., and Bernkop-Schnürch, A. (2014). Pre-systemic metabolism of orally administered drugs and strategies to overcome it. J. Control. Release 192, 301-309. doi: 10.1016/j.jconrel.2014.08.004

Edmondson, R., Broglie, J. J., Adcock, A. F., and Yang, L. (2014). Threedimensional cell culture systems and their applications in drug discovery and cell-based biosensors. Assay Drug Dev. Technol. 12, 207-218. doi: 10.1089/adt.2014.573

Engen, P. A., Green, S. J., Voigt, R. M., Forsyth, C. B., and Keshavarzian, A. (2015). The gastrointestinal microbiome: alcohol effects on the composition of intestinal microbiota. Alcohol Res. 37, 223-236.

Esch, M., King, T., and Shuler, M. (2011). The role of body-on-a-chip devices in drug and toxicity studies. Annu. Rev. Biomed. Eng. 13, 55-72. doi: 10.1146/annurev-bioeng-071910-124629

Esch, M. B., Mahler, G. J., Stokol, T., and Shuler, M. L. (2014). Body-on-a-chip simulation with gastrointestinal tract and liver tissues suggests that ingested nanoparticles have the potential to cause liver injury. Lab Chip 14, 3081-3092. doi: 10.1039/C4LC00371C

Esch, M. B., Sung, J. H., Yang, J., Yu, C., Yu, J., March, J. C., et al. (2012). On chip porous polymer membranes for integration of gastrointestinal tract epithelium with microfluidic 'body-on-a-chip'devices. Biomed. Microdevices 14, 895-906. doi: 10.1007/s10544-012-9669-0

Günzel, D., and Yu, A. S. (2013). Claudins and the modulation of tight junction permeability. Physiol. Rev. 93, 525-569. doi: 10.1152/physrev.00019.2012

Haas, M. K., Berndt, A., Stiller, K. J., Hyckel, P., and Kosmehl, H. (2001). A comparative quantitative analysis of laminin-5 in the basement membrane of normal, hyperplastic, and malignant oral mucosa by confocal immunofluorescence imaging. J. Histochem. Cytochem. 49, 1261-1268. doi: 10.1177/002215540104901008

Huh, D., Hamilton, G. A., and Ingber, D. E. (2011). From 3D cell culture to organs-on-chips. Trends Cell Biol. 21, 745-754. doi: 10.1016/j.tcb.2011.09.005

Imparato, G., Urciuolo, F., Casale, C., and Netti, P. A. (2013). The role of microscaffold properties in controlling the collagen assembly in $3 \mathrm{D}$ dermis equivalent using modular tissue engineering. Biomaterials 34, 7851-7861. doi: 10.1016/j.biomaterials.2013.06.062

Inamdar, N. K., and Borenstein, J. T. (2011). Microfluidic cell culture models for tissue engineering. Curr. Opin. Biotechnol. 22, 681-689. doi: 10.1016/j.copbio.2011.05.512

Lee, S. Y., and Sung, J. H. (2018). Gut-liver on a chip toward an in vitro model of hepatic steatosis. Biotechnol. Bioeng. 115, 2817-2827. doi: 10.1002/bit.26793

Mahler, G. J., Esch, M. B., Glahn, R. P., and Shuler, M. L. (2009). Characterization of a gastrointestinal tract microscale cell culture analog used to predict drug toxicity. Biotechnol. Bioeng. 104, 193-205. doi: 10.1002/bit.22366

Maurice, C. F., Haiser, H. J., and Turnbaugh, P. J. (2013). Xenobiotics shape the physiology and gene expression of the active human gut microbiome. Cell 152, 39-50. doi: 10.1016/j.cell.2012.10.052

Mutlu, E. A., Gillevet, P. M., Rangwala, H., Sikaroodi, M., Naqvi, A., Engen, P. A., et al. (2012). Colonic microbiome is altered in alcoholism. Am. J. Physiol. Gastrointest. Liver Physiol. 302, G966-G978. doi: 10.1152/ajpgi.00380.2011

Prot, J. M., Maciel, L., Bricks, T., Merlier, F., Cotton, J., Paullier, P., et al. (2014). First pass intestinal and liver metabolism of paracetamol in a 
microfluidic platform coupled with a mathematical modeling as a means of evaluating ADME processes in humans. Biotechnol. Bioeng. 111, 2027-2040. doi: 10.1002/bit.25232

Rowland, M. (1972). Influence of route of administration on drug availability. J. Pharm. Sci. 61, 70-74. doi: 10.1002/jps.2600610111

Sambuy, Y., De Angelis, I., Ranaldi, G., Scarino, M., Stammati, A., and Zucco, F. (2005). The caco-2 cell line as a model of the intestinal barrier: influence of cell and culture-related factors on caco- 2 cell functional characteristics. Cell Biol. Toxicol. 21, 1-26. doi: 10.1007/s10565-005-0085-6

Santaguida, S., Janigro, D., Hossain, M., Oby, E., Rapp, E., and Cucullo, L. (2006). Side by side comparison between dynamic versus static models of blood-brain barrier in vitro: a permeability study. Brain Res. 1109, 1-13. doi: 10.1016/j.brainres.2006.06.027

Sibilio, S., De Gregorio, V., Urciuolo, F., Netti, P. A., and Imparato, G. (2019). Effect of peristaltic-like movement on bioengineered intestinal tube. Mater. Today Bio. 4:100027. doi: 10.1016/j.mtbio.2019.100027

Singer, M. V., and Brenner, D. (2006). Alcohol and the Gastrointestinal Tract. Basel: Karger Medical and Scientific Publishers.

Tsamandouras, N., Chen, W. L. K., Edington, C. D., Stokes, C. L., Griffith, L. G., and Cirit, M. (2017). Integrated gut and liver microphysiological systems for quantitative in vitro pharmacokinetic studies. AAPS J. 19, 1499-1512. doi: 10.1208/s12248-017-0122-4
Valatas, V., and Kolios, G. (2009). Ethanol effects on mucin glycosylation: another kick in the gut? Ann. Gastroenterol. 22, 138-140.

van Midwoud, P. M., Merema, M. T., Verpoorte, E., and Groothuis, G. M. (2010). A microfluidic approach for in vitro assessment of interorgan interactions in drug metabolism using intestinal and liver slices. Lab Chip 10, 2778-2786. doi: $10.1039 / \mathrm{c} 01 \mathrm{lc} 00043 \mathrm{~d}$

Weise, F., Fernekorn, U., Hampl, J., Klett, M., and Schober, A. (2013). Analysis and comparison of oxygen consumption of HepG2 cells in a monolayer and three-dimensional high density cell culture by use of a matrigrid@. Biotechnol. Bioeng. 110, 2504-2512. doi: 10.1002/bit.24912

Conflict of Interest: The authors declare that the research was conducted in the absence of any commercial or financial relationships that could be construed as a potential conflict of interest.

Copyright (c) 2020 De Gregorio, Telesco, Corrado, Rosiello, Urciuolo, Netti and Imparato. This is an open-access article distributed under the terms of the Creative Commons Attribution License (CC BY). The use, distribution or reproduction in other forums is permitted, provided the original author(s) and the copyright owner(s) are credited and that the original publication in this journal is cited, in accordance with accepted academic practice. No use, distribution or reproduction is permitted which does not comply with these terms. 